UCRL-ID-128908

\title{
Mechanical Failure Characterization of Optical Components Caused by Laser Induced Damage Initiated at Contaminants
}

\author{
Douglas R. Faux
}

December 1997

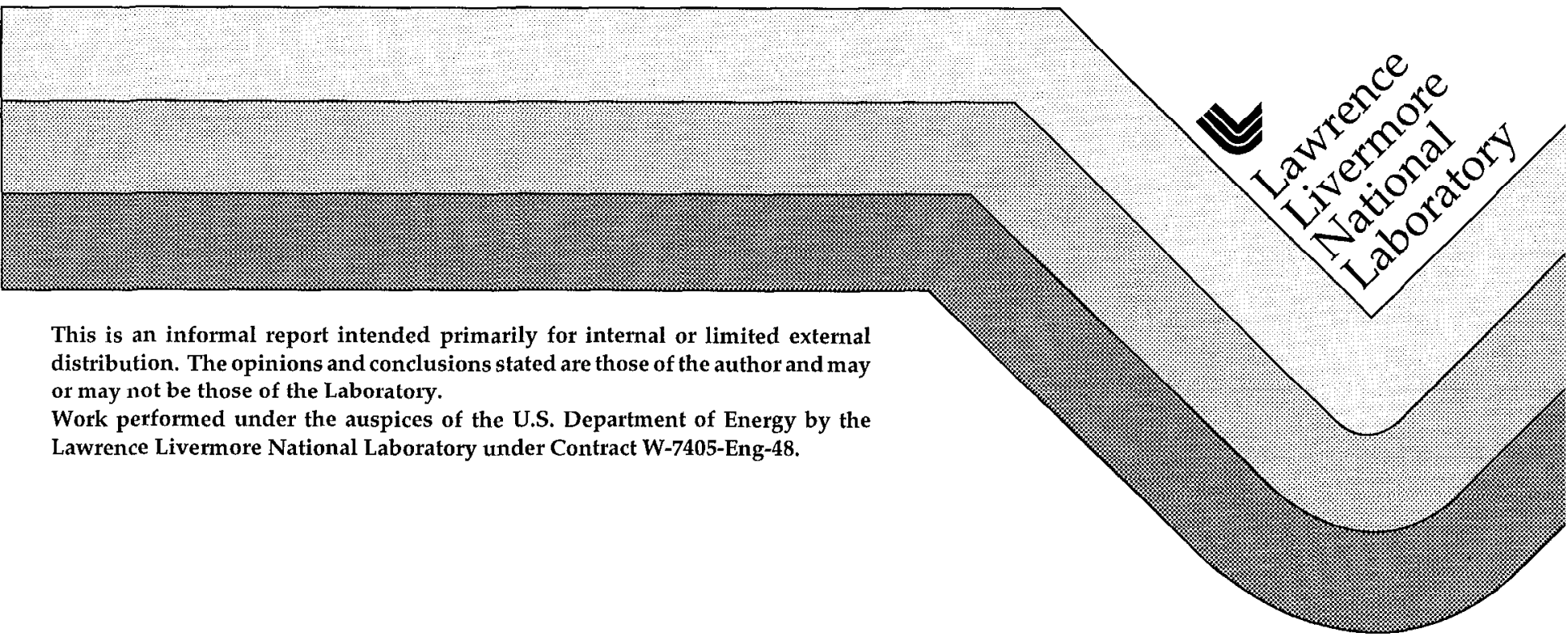




\section{DISCLAIMER}

This document was prepared as an account of work sponsored by an agency of the United States Government Neither the United States Government nor the University of California nor any of their employees, makes any warranty, express or implied, or assumes any legal liability or responsibility for the accuracy, completeness, or usefulness of any information, apparatus, product, or process disclosed, or represents that its use would not infringe privately owned rights. Reference herein to any specific commercial product, process, or service by trade name, trademark, manufacturer, or otherwise, does not necessarily constitute or imply its endorsement, recommendation, or favoring by the United States Government or the University of California The views and opinions of authors expressed herein do not necessarily state or reflect those of the United States Government or the University of California, and shall not be used for advertising or product endorsement purposes.

This report has been reproduced directly from the best available copy

Available to DOE and DOE contractors from the Office of Scientific and Technical Information

P.O. Box 62, Oak Ridge, TN 37831

Prices available from (615) 576-8401, FTS 626-8401

Available to the public from the National Technical Information Service

U.S. Department of Commerce 5285 Port Royal Rd., Springfield, VA 22161 


\title{
Mechanical Failure Characterization of Optical Components Caused by Laser Induced Damage Initiated at Contaminants ${ }^{1}$
}

\author{
Douglas R. Faux
}

October, 1997

\begin{abstract}
The goal of this research is to quantify by numerical techniques the effects of surface and subsurface absorbing defects on damage initiation and growth in high power laser optical components. The defects include laser absorbing spots (e.g., surface particulate contamination) and surface damage regions (e.g., micro-cracks and voids) which are present due to environmental exposure and fabrication processes. This report focuses on three sources of contamination that can cause damage to optical components: 1) Front surface particle contamination, 2) Back surface particle contamination, and 3) Subsurface particle contamination. The DYNA2D (non-linear structural mechanics) code was used to model the growth of damage in the glass substrate.

The damage in the nominally transparent glass substrate as a result of front surface particle contamination was found to be dependent on the magnitude of the resultant pressure pulse applied to the particle and the initial area of contact between the particle and glass substrate. The pressures generated from a back surface particle being blown off the surface provided sufficient loading to severely damage (crack) the glass substrate. A subsurface Ceria dioxide particle showed a strong surface interaction that influenced the formation and direction of the damage (cracking) that ultimately resulted in the blow-out of the damaged material leaving a relatively clean crater in the glass. Crater shape and size was determined.

Since fused silica is the most transparent, and therefore laser damage resistant, of the optical materials, it is used for the most at-risk optical elements. The present studies are for a fused silica substrate.

Some oxides such as Ceria are transparent in the infra-red and visible, but absorbing in the UV part of the spectrum. Because ICF lasers like NIF use frequency tripling, effects of such oxides must be included.
\end{abstract}

\footnotetext{
${ }^{1}$ This work was supported by Lawrence Livermore National Laboratory LDRD/ERD funding, project 97 END-011.
} 
Table of Contents

page

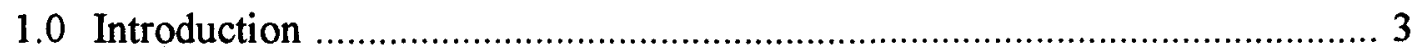

2.0 Mechanical Properties and Failure Characteristics of Fused Silica .............. 3

3.0 DYNA2D Bulk Viscosity and Spatial Zone Requirements........................ 5

3.1 DYNA2D Brittle Damage Models ..................................................... 6

4.0 Front Side Particle Contamination ..................................................... 7

4.1 Front Side Particle Contamination: One-Dimensional Spallation ................... 7

4.2 Front Side Particle Contamination: Two-Dimensional Spallation ................. 9

5.0 Back Side Particle Contamination .................................................. 12

6.0 Subsurface Particle Contamination: Cerium Oxide ................................. 13

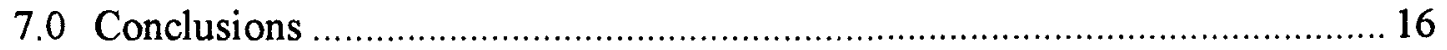

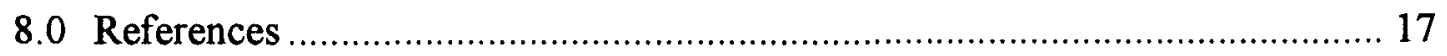

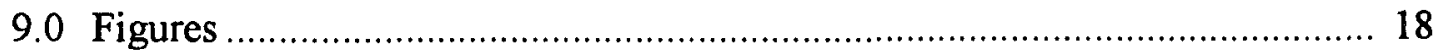




\subsection{Introduction}

The Beamlet spatial filter input window failure caused significant damage to the laser and a month of downtime. Experimental evidence indicates that particulate contamination on the lens surface or embedded below the surface may have been the precursor to failure. A failure scenario involves a particle on the back surface being irradiated by laser light, creating a hot expanding plasma, which then imparts a severe pressure loading on the glass surface creating micro-cracks which can grow to unacceptable damage.

The goal of this research is to quantify by numerical techniques the effects of surface and subsurface defects on damage initiation and growth in high power laser optical components. The defects include laser absorbing spots (e.g., surface particulate contamination) and surface damage regions (e.g., micro-cracks and voids) which are present due to environmental exposure and fabrication processes. The damage initiation process is described in terms of the stress waves and thermal stresses which develop when intense laser radiation is absorbed by foreign material attached to the optical surface producing rapid local heating and material evaporation and ablation. The failure and damage growth in the optical components is described using the concepts of continuum mechanics.

This report concentrates on three sources of contamination that can cause damage to optical components: 1) Front surface particle contamination, 2) Back surface particle contamination, and 3) Subsurface particle contamination. Understanding the precursor to damage by various mechanisms will lead to surface cleanliness and surface finish design requirements.

\subsection{Mechanical Properties and Failure Characteristics of Fused Silica}

To perform detailed analyses of the events that lead up to failure and fracture of the glass substrate, the dynamic properties of the material must be well understood. For ICF revelant cases, events are occurring on a nanoseconds time scale. Temperatures and pressures in the plasma and adjacent glass material may reach $1 \mathrm{eV}(11,605 \mathrm{~K})$ and up to 150 Kbars.

Shock waves comprise an integral part of the study of dynamic behavior of material under high pressure. Equations governing the behavior of shock waves are derived by applying the laws of conservation of mass, momentum, and energy. The equations developed by applying these laws are referred to as the Hugoniot equations. The Hugoniot curve is a material property which is a locus of attainable shock states and is analogous to a stress-strain curve in uniaxial stress. The wave velocity is constant up to the Hugoniot Elastic Limit (HEL) above which the slope increases with stress.

Typical shock compression curves are defined in Figure 1, illustrating a perfect elasto-plastic solid, a quasi elasto-plastic solid, an elasto-isotropic solid and an isotropic static compression curve. For the perfect elasto-plastic solid, the material preserves almost constant offset from the isotropic static curve. This offset corresponds to $(2 / 3) Y$, where $Y$ is the yield stress. For the quasi elasto-plastic solid, the material loses some shear strength but still preserves considerable shear strength in the plastic region. Most metals 
are described as elasto-plastic materials. For the elasto-isotropic solid, the material catastrophically loses almost all shear strength, and the compression curve exhibits a large negative curvature above the HEL and immediately approaches the isotropic static curve. The small offset is due to the temperature increase. Most all brittle materials behave as an elasto-isotropic material, including $\mathrm{Si}^{4}$ and $\mathrm{SiO}_{2}$.

An equation of state (EOS) may be derived from a Hugoniot curve. Wackerle ${ }^{1}$ performed plane wave experiments on high purity $\mathrm{SiO}_{2}$ free of visible flaws. $\mathrm{SiO}_{2}$ displays a cusp in its Hugoniot at $9.8 \mathrm{GPa}$, above which is a region of high compressibility, followed at somewhat higher pressures by considerable stiffening of the material. A tri-linear fit was needed of the shock and particle velocity curve to accurately represent the material. Sugiura ${ }^{2}$ determined an HEL of $8.81 \mathrm{GPa}$ for fused quartz (T1000, Toshiba Ceramics Co. Ltd.). The elastic region was nearly reversible; however it was noticed that the compressibility did increase with pressure. In shock loading, a deviatoric stress of $7.01 \mathrm{GPa}$ exists before yielding. Grady ${ }^{3}$ performed spall experiments on fused silica and found no spall for a tensile stress of $3.1 \mathrm{GPa}$. Barker [private communication] observed no spall for a tensile stress of approximately $1.3 \mathrm{GPa}$. Figure 2 illustrates the equation of state data for "low" pressure, "high" pressure and a constant bulk modulus assumption. If we assume that the pressure in an element stays less than $200 \mathrm{Kbars}$, then the constant bulk modulus or "low" pressure EOS should suffice. If pressures go above $200 \mathrm{Kbars}$, then a modified EOS accounting for high pressure response would be needed. The measured HEL is related to the equivalent dynamic compressive strength $\left(Y_{c}\right)$ through the relation,

$$
\mathrm{HEL}=\frac{1-v}{1-2 v} \mathrm{Y}_{\mathrm{c}}
$$

where $v$ is the Poisson's Ratio. This relation is obtained by considering the elastic relation between the longitudinal stress and the lateral stress behind the shock front and the Tresca yield criterion. This relation works quite well for shock loaded metals. For brittle materials, if the Griffith yield criterion is used, the relationship between HEL and $Y_{c}$ becomes (Rosenberg ${ }^{4}$ ),

$$
\text { HEL }=\frac{1-v}{(1-2 v)^{2}} Y_{c}
$$

The Griffith yield criterion may also be applied to spall data yielding,

$$
\mathrm{HEL}=\frac{1-v}{(1-2 v)^{2}} 8 \sigma_{\text {spall }}
$$

Applying the above equations and assuming a HEL of $9.5 \mathrm{GPa}$ and $v$ of 0.17 , yields $Y c=$ $4.98 \mathrm{GPa}$ and $\sigma_{\text {spall }}=0.623 \mathrm{GPa}$. Table 1 summarizes the static and dynamic properties of fused silica glass. 
Table 1: Static and Dynamic Properties of Fused Silica

\begin{tabular}{|l|c|}
\hline \multicolumn{1}{|c|}{ Property } & Value \\
\hline Young's Modulus (GPa) & 73.77 \\
\hline Shear Modulus (GPa) & 31.53 \\
\hline Bulk Modulus (GPa) & 37.26 \\
\hline Poisson's Ratio & 0.17 \\
\hline Static Tensile Strength (MPa) & $49-59$ \\
\hline Static Compressive Strength (MPa) & $1110-1170$ \\
\hline Hugoniot Elastic Limit (GPa) & $8.8-10$ \\
\hline Dynamic Compressive Strength (GPa) & $5^{\mathrm{a}}-7$ \\
\hline Dynamic Spall Strength (GPa) & $0.62^{\mathrm{a}}, 1.3-3.1^{\mathrm{b}}$ \\
\hline
\end{tabular}

Under relatively small loading, brittle material fails when the maximum tensile stress reaches a certain criterion; however, under large dynamic loading, brittle material can fracture in compression. The compressive failure is complex and depends on the stress state. When lateral confinement is absent, micro-cracks nucleate at the tips of existing flaws, and these cracks are found to grow under the influence of local tensile stresses around these cracks, leading to a splitting type of failure. When confined, brittle materials fail under faulting. Faulting is a type of compressive failure in which the nucleated micro-cracks grow and coalesce at some angle to the axial direction. In most cases compressive failure does not occur unless the stress state is above the HEL.

Certain brittle materials (soda-lime, Pyrex and K19 glass) fail under compression in the region behind the shock front even when stresses are below the HEL. This failure front can propagate behind a compressive shock front at approximately the shear wave velocity and is dependent on impact velocity. The failure wave velocity also decreases with distance. Experiments with short load pulses have shown that unloading will arrest the failure wave propagation. This failure wave may account for the evolution of tension cracks (at the tips of pre-existing micro-cracks) under compression of brittle solids. There is a dramatic lowering of the shear strength behind the failure fronts due to pulverization of the glass (Kanel ${ }^{5}, \mathrm{Brar}^{6}$, Bourne $^{7}$ ).

\subsection{DYNA2D Bulk Viscosity and Spatial Zone Requirements}

Numerical hydrocodes employ artificial bulk viscosity methods to eliminate shock discontinuities by spreading the shock front over several elements. In applying artificial viscosity, the pressure in compressing elements is augmented by an artificial viscous term, $q$, before evaluating the stress divergence. Away from shocks, the $q$ term is negligible leaving the solution relatively unperturbed. Across the shock, the Hugoniot jump conditions are valid. Improper element size or inadequate artificial viscosity may cause diffusion or dispersion of the shock front. DYNA2D employs two types of artificial viscosity, "standard DYNA2D" and "Richards-Wilkins." Both of these methods utilize a linear and quadratic term which are input parameters. The Richards-Wilkins 
viscosity method contains an additional term which is used to prevent diffusion losses in converging geometries.

One-dimensional plane strain calculations were performed to determine the element size required to track a short duration pressure pulse and determine the appropriate amount of artificial viscosity to use. A 10 nanosecond $10 \mathrm{Kbars}$ full width half maximum (FWHM) Gaussian pressure pulse was applied to one end of the onedimensional bar. The pressure wave travels down the bar and its peak magnitude and period is tracked. Figure 3 shows a plot of the maximum peak pressure as a function of distance traveled down the bar for different bulk viscosity methods and coefficients. A 2 microns zone size is used for these calculations. Based on this analysis the RichardWilkins viscosity method with a quadratic coefficient of 1.5 and a linear coefficient of 0.0033 minimizes any energy loss. Figure 4 shows a plot of the maximum peak pressure as a function of distance traveled down the bar for different element resolutions. A 1 to 2 microns zone size is required to accurately track the 10 nanosecond pressure pulse. Zone size requirement also depends on how far the shock front must travel. The farther the shock front must travel the more susceptible it is to diffusion losses. Mesh resolution and viscosity terms need to be addressed when setting-up any finite element analysis.

\subsection{DYNA2D Brittle Damage Models}

Four brittle damage models were incorporated into DYNA2D for the use of modeling dynamic impact events with brittle materials such as ceramics and glasses. Three of the brittle damage models implemented use a scalar damage parameter to track cumulative damage while the fourth brittle damage model incorporates a tensor damage via the explicit tracking of $3 \mathrm{D}$ cracks within an element.

For the three scalar damage models, the constitutive response of undamaged material is treated with either an Isotropic-Elastic-Plastic or a Steinberg-Guinan High Rate Elastic Plastic flow rule. Failed material is treated with a piece-wise linear definition of yield stress versus pressure. The transition between undamaged and fully damaged material is accomplished through the evolution of the scalar damage parameter, $D$. The three scalar damage models differ in their evolution equations for $D$. The shear modulus, yield strength and pressure are transitioned between the undamaged values and fully damaged values as $D$ goes from 0 -1. The three scalar damage models are Modified TulerButcher Damage Model, Modified Cagnoux-Glenn Brittle Damage Model, and SteinbergTipton Brittle Damage Model .

The tensor damage model incorporated into DYNA2D to calculate the brittle damage response of glass is $A$ Continuum Tensile Failure Model With Friction. Fracture is modeled in a continuum sense by introducing a symmetric tensor $\mathrm{e}^{\mathrm{v}}$ which denotes void strain. The void strain is determined by restricting the traction vector that is applied on a fracture surface. The model allows the formation of up to three orthogonal fracture surfaces, which are normal to the right-handed orthonormal set of unit vectors characterizing the fracture triad. Compressive yielding and tensile failure are each treated separately. Compressive failure involves the evolution of a scalar damage variable and tensile failure involves the formation of three fracture surfaces and the evolution of a void strain. 
A complete description of these models and their applications to ceramic and glass damage can be found in [8].

\subsection{Front Side Particle Contamination}

If a highly absorbing metal particle is on the front side of an optic and is hit with a laser, a pressure pulse due to the laser light absorption on the surface of the particle will be transmitted through the particle. If the particle is not flush against the optics (free surface) spallation may occur. The spallation is due to the crossing of two rarefaction waves. One is coming from the front of the plate when the loading falls off, and the other is generated from the rear surface, when the incident shock reflects back into the material. If the tensile conditions are sufficient in magnitude and time application, they can lead to the formation of a spall plane. The intent of the following section is to investigate the pressure required to cause spallation in an aluminum bar that represents front surface contamination through the use of one-dimensional plane strain analysis.

\subsection{Front Side Particle Contamination: One-Dimensional Spallation}

An aluminum bar 100 microns long, was modeled as a continuum with a SteinbergGuinan constitutive law and a Mie-Gruneisen equation of state. The constitutive law accounts for high strain rate behavior with pressure hardening and thermal softening. The Tuler-Butcher ${ }^{9}$ ductile failure model was chosen to model the spallation since it includes time dependent failure. The validity of this failure model for short duration (600 ps to 25 $\mathrm{ns})$ loading has been proven experimentally by Boustie et al. ${ }^{10}$ The material parameters $\sigma_{0}, A$ and $K$ for aluminum 6061-T6 are from [9] and shown in Table 2. The SteinbergGuinan material parameters and Mie-Gruneisen parameters were obtained from [11].

Table 2: Aluminum 6061-T6 Tuler-Butcher Parameters

\begin{tabular}{|l|l|}
\hline Density & $2.768 \mathrm{gm} / \mathrm{cm}^{3}$ \\
\hline$\sigma_{0}$ & $0.01 \mathrm{Mbar}$ \\
\hline $\mathrm{A}$ & 2.02 \\
\hline $\mathrm{K}$ & $22.9 \mathrm{e}-6 \mathrm{Mbar}^{2.02}-\mu \mathrm{s}$ \\
\hline
\end{tabular}

A Gaussian FWHM pressure pulse was applied to one end of a 100 microns long aluminum bar with the other end free. The purpose of these calculations was to determine what pressure, Pmax at various pulse widths, is required to just cause spallation in the aluminum bar. Figure 5 illustrates the five pulse widths used in this investigation 1,2,4,8 and 16 nanoseconds FWHM Gaussian pulses. Figure 6 shows the results of the study. The solid curve shows the minimum pressure, Pmax, required for a given pulse width to cause spallation in the aluminum bar. The dashed curve shows the corresponding spall plane thickness, the amount of material ejected. As a point of reference, a Pmax of 25 $\mathrm{Kbars}$ is required to cause spallation for a long duration pressure pulse. The dramatic 
effect of time dependency on the spallation of aluminum at short pulse widths is demonstrated. Spallation in a one-dimensional aluminum bar is dependent on the applied maximum pressure and the period of the Gaussian pulse. Equation 4 represents the fit to the data of Figure 6, (Kbars,nanoseconds).

$$
P_{\max }=384.72 \tau^{-0.5933}
$$

The $150 \mathrm{Kbars} 4$ nanosecond Gaussian pulse case will be used to discuss in more detail the one-dimensional results. Figure 7 is a line drawing of the pressure in the bar at 15 nanoseconds. The pressure pulse is traveling left to right and has not reached the free end at the right. An elastic precursor is visible in front of the plastic wave. The material has undergone significant yielding, $16 \%$ effective plastic strain (yield strength for aluminum is $2.9 \mathrm{Kbars}$ ). Once the initial compressive pulse reaches the free end, a tensile rarefaction wave is formed and travels right to left. This tensile wave begins to damage the material and may cause spallation based on the Tuler-Butcher criteria. When an element has reached the critical spall condition, $D \geq 1$, it is deleted from the analysis. Figure 8 is a line drawing of the damage variable, $D$, versus distance along the aluminum bar at 40 nanoseconds. A significant portion of the bar is damaged and has undergone void growth and a spall plane formed at 26 microns from the end of the bar. The ejected material has a velocity of $0.018 \mathrm{~cm} / \mu \mathrm{s}$.

When an irregular shaped particle (aluminum) is on the front surface of the optic, there are surfaces which are and are not in contact with the optic. This presents the question, how far off the surface must the particle be to generate sufficient tensile stress to cause spallation? To answer this, one can look at the free end displacement of the aluminum bar. For the 150 Kbars 4 nanoseconds pressure pulse, spallation occurs at 32 nanoseconds representing a tip displacement of 8.8 microns. Therefore, if the aluminum particle is within 8.8 microns of the optic, a portion of the initial compressive wave is transmitted into the optic and is not available to form a tensile rarefraction wave which is necessary for spallation in the aluminum particle. The transfer of pressure across a material boundary is dependent on the impedance match between the two materials. Figure 9 is a graph of the minimum distance the leading edge of a front surface particle must be to the optic for spallation to occur for various pulse widths.

To apply the one-dimensional spall methodology to an actual laser absorption calculation, Rubenchik ${ }^{12}$ modeled an 8.5 nanosecond $40 \mathrm{~J} / \mathrm{cm}^{2}$ pulse on a 200 microns aluminum slab. The laser energy is absorbed in a thin skin depth. In a narrow boundary layer (less than 1 micron) the temperature is about $2 \mathrm{eV}$, high enough to produce a flash of light. After a short transient, a well formed pressure pulse (Figure 10) moves through the material at a characteristic velocity. This pulse is about 25 microns long with a duration slightly less than that of the laser pulse. The peak pressure is about $70 \mathrm{Kbars}$. At longer times, the vapor remains hot, but the aluminum is cool since thermal conduction times are long compared to shock traversal times. This pressure pulse was applied to a 100 microns long aluminum bar modeled with the Steinberg-Guinan constitutive law, MieGruneisen equation of state and the Tuler-Butcher ductile failure criteria. Figure 11 is a line drawing of the damage variable, $D$ versus distance along the aluminum bar. There is not sufficient enough damage to generate a spall plane. It is important to note that if a 
simple spall criteria (spall occurs when the most principle stress reaches the spall stress) would have been used, the analysis would have predicted a spall plane at approximately 10 microns from the free end.

\subsection{Front Side Particle Contamination: Two-dimensional Spallation}

An aluminum spherical particle, 100 microns in diameter, was placed in contact with a $\mathrm{SiO}_{2}$ substrate. Initial contact was assumed to be a $40^{\circ}$ apex angle. $\mathrm{A} 150 \mathrm{Kbars} 4$ nanosecond FWHM Gaussian pressure pulse was applied to the surface of the sphere with a cosine squared ${ }^{13}$ pressure distribution, Figure 12. The Steinberg-Guinan constitutive law, Mie-Gruneisen equation of state and the Tuler-Butcher failure criteria for spall was used to model the behavior of the aluminum particle (refer to section 4.1 for aluminum material parameters). The $\mathrm{SiO}_{2}$ substrate was modeled with the Modified Rubin/Attia 3D Crack Damage Model ${ }^{8}$ and a Linear Polynomial equation of state. Table 3 shows the constitutive parameters used for the substrate and Table 4 shows the EOS parameters used for the substrate.

Table 3: 3D Crack Model Parameters for Fused Silica

\begin{tabular}{|l|c|}
\hline Parameters & Value \\
\hline G (Mbar) & 0.3152 \\
\hline Y (Mbar) & 0.0525 \\
\hline Shear Fail Limit & 0.1 \\
\hline Tensile Limit & 0.00546 \\
\hline Friction & 0.3 \\
\hline Shear Degrade & 0.333 \\
\hline failure slope & 0.8 \\
\hline sigf (Mbar) & 0.030 \\
\hline
\end{tabular}

Table 4: Linear Polynomial EOS Parameters for Fused Silica

\begin{tabular}{|l|c|}
\hline \multicolumn{1}{|c|}{ Parameter } & Value \\
\hline C0 (Mbar) & 0 \\
\hline C1 (Mbar) & 0.4167 \\
\hline C2 (Mbar) & -0.1748 \\
\hline C3 (Mbar) & 0.0405 \\
\hline C4 & 0.039 \\
\hline C5 & 0.0 \\
\hline C6 & 0.0 \\
\hline E0 (Mbar-cc/cc) & 0.0 \\
\hline V0 & 1.0 \\
\hline
\end{tabular}

Figures 13 and 14 show the progression of pressure in the aluminum particle at $10,15,20$, and 25 nanoseconds, respectively. An initial compressive wave followed by a tensile wave occurs in the aluminum particle. This tensile wave causes the center region of 
the particle to fail. The initial compressive wave reflects off the aluminum/fused silica surface due to an impedance mismatch, and forms a tensile wave. This tensile wave superimposes on the existing tensile wave and forms the lower failed region. Two distinct failure regions develop in the aluminum particle from tensile loading, Figure 15. The aluminum particle has undergone severe plastic deformation as evident from a plot of the effective plastic strain, Figure 16.

Damage in the glass substrate will be shown by displaying an element damage state. Fringe values of $-1,-2$ or -3 indicate the number of tensile cracks in an element. A fringe value of 1 means undamaged material and a value of 2 means compressive yielding. The damage in the glass begins at 20-22 nanoseconds at the outer contact points where shear stresses develop first, Figure 17. The damage grows into the substrate then connects to the subsurface damage ( 12 microns below the surface) where the shear stresses reach their maximum, Figure 18. Damage then continues to expand out in a semi-circular fashion with visible fingers of damage emanating from the main subsurface damage region, Figure 19. The aluminum particle separates from the substrate at 24 nanoseconds when the tensile wave reaches the interface and the gap grows until the damaged glass expands and fills the gap. Significant damage of the glass occurs to a depth of 40 microns with fingers of damage growing to 80 microns deep. The diameter of damage is 70 microns.

The previous analysis was repeated, but with a 100 Kbars 4 nanosecond FWHM Gaussian pressure pulse. Figure 21 shows the damage in the glass at 50 nanoseconds. Significantdamage of the glass occurs to a depth of 22 microns with fingers of damage growing to 42 microns deep. Radial cracking is not as apparent for the $100 \mathrm{Kbars}$ case. The diameter of damage is 60 microns. When the pressure pulse was reduced to $75 \mathrm{Kbars}$, slight damage ( 5 microns deep) was observed in the glass directly under the aluminum particle. The spall plane at 12 microns deep was just beginning to form. The maximum pressure of the Gaussian pulse does not indicate the pressure loading the glass substrate experiences Table 5 shows the pressure applied to the sphere, the pressure in the aluminum at the aluminum/glassinterface and the pressure in the glass substrate at the interface. The large reduction in pressure by the time the compressive wave reaches the interface is due to yielding and failure in the aluminum sphere.

Table 5: Pressures in the Aluminum and Glass Substrate

\begin{tabular}{|c|c|c|}
\hline $\begin{array}{c}\text { Pressure } \\
\text { Applied } \\
\text { (Kbars) }\end{array}$ & $\begin{array}{c}\text { Pressure in Al } \\
\text { at Interface } \\
\text { (Kbars) }\end{array}$ & $\begin{array}{c}\text { Pressure in glass } \\
\text { at interface } \\
\text { (Kbars) }\end{array}$ \\
\hline 150 & 68 & 30 \\
\hline 100 & 35 & 17 \\
\hline 75 & 18 & 10 \\
\hline 50 & 10 & 7 \\
\hline
\end{tabular}

Two additional analyses were performed to investigate initial aluminum particle/glass contact angle. Figure 22 shows the damage in the glass for a $150 \mathrm{Kbars} 4$ nanosecond FWHM Gaussian pressure pulse with an initial angular contact of $20^{\circ}$. Significant damage of the glass occurs to a depth of 20 microns with fingers of damage growing to 45 microns deep. The diameter of damage is 40 microns. Figure 23 shows the 
damage in the glass for a 150 Kbars 4 nanosecond FWHM Gaussian pressure pulse with an initial angular contact of $0^{\circ}$. Significant damage of the glass occurs to a depth of 30 microns. The finger-like cracking is not well defined. The diameter of damage is 40 microns near the surface and gets smaller as the damage grows into the substrate.

To investigate the damage model response, a final analysis of the aluminum particle on a glass substrate was performed using the Modified Cagnoux-Glenn Damage Model for the glass substrate. The parameters for the Modified Cagnoux-Glenn Damage Model are shown in Table 6. An aluminum spherical particle, 100 microns in diameter, was placed in contact with $\mathrm{SiO}_{2}$ substrate. Initial contact was assumed to be a $40^{\circ}$ apex angle. A 150 Kbars 4 nanosecond FWHM Gaussian pressure pulse was applied to the surface of the sphere with a cosine squared pressure distribution.

Table 6: Modified Cagnoux-Glenn Parameters for Glass Substrate

\begin{tabular}{|l|l|}
\hline Parameters & Value \\
\hline sigc (Mbar) & 0.0039 \\
\hline B (Mbar-us) & $7.0 \mathrm{e}-10$ \\
\hline b (Mbar $\left.{ }^{-1}\right)$ & 700 \\
\hline ffail & 0.9 \\
\hline feta & 1.0 \\
\hline fbulk & 1.0 \\
\hline failure slope & 0.8 \\
\hline sigf (Mbar) & 0.030 \\
\hline
\end{tabular}

Figure 24 shows the damage in the glass substrate at 50 nanoseconds. The color red signifies that the element has completely failed. Finger-like cracks extend from the main damage region. Significant damage of the glass occurs to a depth of 45 microns with fingers of damage growing to 90 microns deep. The diameter of damage is 70 microns. Similar results are observed as compared to Figure 20, indicating that the damage in the glass substrate is not too sensitive to the brittle damage model used. Table 7 tabulates the results of each analysis.

Table 7: Aluminum Particle on Glass Substrate Analysis Results

\begin{tabular}{|c|c|c|c|c|}
\hline & \multicolumn{4}{|c|}{ Applied Pressure Pulse (Kbars) } \\
\hline Contact Angle $\left(^{\circ}\right)$ & 75 & 100 & 150 & $150^{*}$ \\
\hline 0 & & & $30,37,40$ & \\
\hline 20 & & & $22,42,40$ & \\
\hline 40 & $5,12,10$ & $22,40,60$ & $40,80,70$ & $45,90,70$ \\
\hline
\end{tabular}

Legend: The first number is the depth of the main damage region, the second number is the depth of the finger-likecracks, and the third number is the diameter of the damage region. *Modified Cagnoux-Glenn Damage Model.

Particle shape was not addressed, but would also play an important role in understanding the tranmission of pressure (loading) into the glass substrate. Irregular 
shaped particles would absorp laser light differently and subsequently load the glass substrate in a non-uniform manner.

\subsection{Back Side Particle Contamination}

Back surface particle contamination of optics components involves a particle on the back surface being irradiated by laser light, creating a hot "confined" expanding plasma, which then imparts a severe pressure loading on the glass surface creating microcracks and unacceptable damage. To determine the pressure loading on the glass surface, LASNEX was used to model the formation of the plasma and its subsequent pressure loading of the glass. A 100 microns diameter X 5 microns thick aluminum disc was placed on the back side of a $\mathrm{SiO}_{2}$ substrate. An $18 \mathrm{~J} / \mathrm{cm}^{3}$ Gaussian Pulse, 3 nanoseconds FWHM was applied to the optic, Figure 25. Pressure as a function of time and radius at the solid-liquid interface $(0.1 \mathrm{eV})$ was obtained from the LASNEX calculation ${ }^{14}$. Figure 26 shows the time history of pressure at the solid-liquid interface at the centerline location (radius $=0$ ). The maximum pressure occurs at 3.52 nanoseconds. Figure 27 shows how pressure varies with radius at time $=3.52$ nanoseconds. A steep drop off in pressures occurs at the outer diameter of the aluminum disc. Figure 28 shows how the solid-liquid interface moves with respect to time. The interface does not begin to move until 3 nanoseconds into the laser pulse and moves into the substrate only 0.86 microns.

To facilitate transfer of pressure data from LASNEX to DYNA2D, a table lookup feature was incorporated into DYNA2D to apply this temporal and spatially varying pressure history to a virgin glass substrate. A 400 microns diameter X 300 microns deep DYNA2D model was generated to apply the pressure histories to the substrate. Nonreflecting boundary conditions were used to simulate a semi-infinite block. A pressure boundary surface from radius $=0$ to 80 microns was specified on the semi-infinite block and the table look-up feature was used to apply the pressures as a function of radius and time. The $\mathrm{SiO}_{2}$ substrate was modeled with the Modified Rubin/Attia 3D Crack Damage Model and a Linear Polynomial equation of state. Table 8 shows the constitutive parameters used for the substrate.

Table 8: 3D Crack Model Parameters for Fused Silica

\begin{tabular}{|l|l|}
\hline Parameters & Value \\
\hline G (Mbar) & 0.3152 \\
\hline Y (Mbar) & 0.0525 \\
\hline Shear Fail Limit & 0.1 \\
\hline Tensile Limit & 0.008 \\
\hline Friction & 0.3 \\
\hline Shear Degrade & 0.333 \\
\hline failure slope & 0.8 \\
\hline sigf (Mbar) & 0.030 \\
\hline
\end{tabular}

Figures 29-32 show the progression of the pressure in the substrate at $6,14,22$, and 30 nanoseconds, respectively. The pressure pulse starts in a rectangular pattern, but 
dispersion causes the pulse to spread in a spherical nature. Damage behind the main pressure pulse causes pressure concentrations to occur. The peak pressure decreases from $22 \mathrm{Kbars}$ to $14 \mathrm{Kbars}$ as the pulse travels 160 microns into the substrate. Figures 33-36 show the progression of damage in the substrate at $14,22,30$, and 60 nanoseconds, respectively. Damage begins at the outside radius of the disc where shear stresses are maximum. Damage follows the pressure pulse then begins to move out in finger-like cracks at $\sim 45^{\circ}$ angles. Damage is measured to a depth of 120 microns with a diameter of 170 microns.

The specification of the Tensile Limit for the damage model does influence the overall size of the damage region and, therefore, a comparison to experimental results would allow for a better determination of the overall damage region. Additional investigation into particle size variations and laser pulse characteristic should be performed.

\subsection{Subsurface Particle Contamination: Cerium Oxide}

Subsurface particle contamination of the optics have been shown to cause unacceptable damage after irradiation ${ }^{15}$. It has been postulated that particula such as cerium oxide, left over from the polishing process, are absorbing energy in the UV spectrum, expanding and causing small craters on the glass surface. DYNA2D was used to investigate this phenomena by explicitly modeling the cerium oxide particle and the glass substrate. Energy was deposited into the particle, absorptivity from literature was $7 \times 10^{4}$ $\mathrm{cm}^{-1}$ at $355 \mathrm{~nm}$, allowing it to expand and damage the glass substrate. It was assumed that the glass around the cerium oxide particle does not absorb light and heat up. To better model the cerium oxide expansion, the Linear Polynomial equation of state was modified to allow for energy dependent bulk modulus and Gruneisen coefficient.

The first cerium oxide particle modeled was a 100 nanometer diameter particle, 300 nanometers deep in a $\mathrm{SiO}_{2}$ substrate, Figure 37. A Linear Polynomial equation of state with energy dependent Bulk Modulus and Gruneisen gamma was used for the cerium oxide particle, Table 9. The $\mathrm{SiO}_{2}$ substrate was modeled with the Modified Rubin/Attia 3D Crack Damage Model and a Linear Polynomial equation of state, Table 10.

Table 9: Equation of State Parameters for Cerium Oxide

\begin{tabular}{|c|c|c|}
\hline $\begin{array}{c}\text { SpecificEnergy } \\
\text { (Mbar-cc/cc) }\end{array}$ & $\begin{array}{c}\text { Bulk Modulus } \\
\text { (Mbar) }\end{array}$ & $\begin{array}{c}\text { Gruneisen } \\
\text { Gamma }\end{array}$ \\
\hline 0.0 & 1.42 & 1.62 \\
\hline 0.042 & 1.42 & 1.72 \\
\hline 0.0844 & 0.71 & 1.82 \\
\hline 0.1 & 0.575 & 2.0 \\
\hline 0.15 & 0.142 & 2.2 \\
\hline 1.0 & 0.142 & 2.2 \\
\hline
\end{tabular}


Table 10: 3D Crack Model Parameters for Fused Silica

\begin{tabular}{|l|l|}
\hline Parameters & Value \\
\hline G (Mbar) & 0.3152 \\
\hline Y (Mbar) & 0.0525 \\
\hline Shear Fail Limit & 0.1 \\
\hline Tensile Limit & 0.005 \\
\hline Friction & 0.3 \\
\hline Shear Degrade & 0.333 \\
\hline failure slope & 0.8 \\
\hline sigf (Mbar) & 0.015 \\
\hline
\end{tabular}

Energy was deposited into the cerium oxide particle at a constant rate for 3 nanoseconds, Figure 38. Figures 39-41 show the pressure in the cerium oxide particle and glass at 1.0, 2.0 , and 3.0 nanoseconds, respectively. Pressure in the cerium oxide particle builds up to approximately $36 \mathrm{Kbars}$ at 1.8 nanoseconds, then the glass begins to damage, and the cerium oxide particle expands and relieves pressure. The glass substrate experiences pressures of $25 \mathrm{Kbars}$ prior to damage. Figures $42-45$ show the progression of damage in the glass at 1.0,1.9, 2.3, and 3.0 nanoseconds, respectively. Damage in the glass substrate will be shown by displaying an element damage state. Fringe values of $-1,-2$ or -3 indicate the number of tensile cracks in an element. A fringe value of 1 means undamaged material, a value of 2 means compressive yielding, and a value of 3 means compressive failure. At 1.0 nanoseconds, the initial compressive wave has reached the upper surface and reflected back as a tensile wave which initiates damage at the $90^{\circ}$ location. At 1.9 nanoseconds, sufficient reflected tensile waves off the surface has created a spall plane near the surface and the damage is growing down toward the particle. At 2.3 nanoseconds the glass around the particle is undergoing compressive failure as the crack is growing out and toward the surface. The surface damage has grown down and converged with the particle. At 3.0 nanoseconds the conical crack $\left(35^{\circ}\right)$ has reached the surface and one would expect the material within the damaged region to be blown off. For a 100 nanometer diameter particle buried 300 nanometers deep the crater that would be formed would measure 1300 nanometers in diameter and 450 nanometers deep. Figure 46 is a fringe of the velocity of the material at 3.0 nanoseconds. The material is being pushed upward and out of the pit region at approximately $150-200$ meters/second.

The second cerium oxide particle modeled was a 100 nanometer diameter particle, 150 nanometers deep in a $\mathrm{SiO}_{2}$ substrate. Energy was deposited into the cerium oxide particle at a constant rate for 3 nanoseconds as above. Figures 47-50 show the progression of damage in the glass at $0.7,1.0,1.3$, and 1.8 nanoseconds, respectively. A similar damage progression as above occurs. Slightly more influence of the surface is evident by the steeper rise of the conical crack toward the surface. Breakout of the crater would occur at approximately 1.3 nanoseconds and would measure 600 nanometers in diameter and 250 nanometers deep. The cerium oxide particle is being over driven since breakout would occur while the laser pulse is still on. Figure 51 is a fringe of the velocity of the material at 1.8 nanoseconds. The material is being pushed upward and out of the pit region at approximately 300-400 nanometers/nanosecond. Table 11 shows the results for the two cerium oxide contamination analyses. 
Table 11: Subsurface Cerium Oxide Contamination Analysis Results

\begin{tabular}{|c|c|c|c|c|}
\hline $\begin{array}{c}\text { Particle } \\
\text { Diameter } \\
(\mathrm{nm})\end{array}$ & $\begin{array}{c}\text { Particle } \\
\text { Depth } \\
(\mathrm{nm})\end{array}$ & $\begin{array}{c}\text { Crater } \\
\text { Depth } \\
(\mathrm{nm})\end{array}$ & $\begin{array}{c}\text { Crater } \\
\text { Surface } \\
\text { Diameter } \\
(\mathrm{nm})\end{array}$ & $\begin{array}{c}\text { Blowoff } \\
\text { Velocity } \\
(\mathrm{m} / \mathrm{s})\end{array}$ \\
\hline 100 & 150 & 250 & 600 & $300-400$ \\
\hline 100 & 300 & 450 & 1300 & $150-200$ \\
\hline
\end{tabular}

Particle size was not addressed, but would also play an important role in understanding the formation of craters in the surface of the glass. For the cases investigated here, the crater was at a depth that surface interaction influenced the formation and direction of the damage (cracking). Additional depths should be looked at to find out where this surface interaction diminishes. In those cases a more accurate definition of the equation of state of the cerium oxide particle is needed to handle the solid-liquid-vapor transition. Also a more accurate energy deposition scheme would be needed.

For the above cases we explicitly modeled the cerium oxide particle and assumed no absorption in the glass. With strong enough absorption, it would be to assume the cerium oxide particle is a seed for absorption to start in the glass. The glass around this seed particle then absorbs energy, expands and causes damage to the surrounding substrate. To analyze this approach, a detailed equation of state would be needed for the $\mathrm{SiO}_{2}$ to handle the solid-liquid-vapor transition along with a detailed energy deposition scheme.

\subsection{Conclusions}

Front surface particle contamination was modeled as an aluminum spherical particle in contact with a glass substrate. Varying pressure pulses were applied to the top surface of the particle to simulate laser absorption. The damage in the glass substrate was found to be dependent on the magnitude of the pressure pulse and the initial area of contact between the aluminum particle and the glass substrate. An applied surface pressure to the aluminum sphere of at least $75 \mathrm{Kbars}$ was required to cause damage in the glass substrate.

Back surface particle contamination of optical components involves a particle on the back surface being irradiated by laser light, creating a hot expanding plasma, which then imparts a severe pressure loading on the glass surface. To determine the pressure loading on the glass surface, LASNEX was used to model the formation of the plasma and its subsequent pressure loading on the glass. A pressure loading of approximately 45 Kbars resulted in severe damage (cracking) of the glass substrate.

Subsurface particle contaminations of optical components have been shown to cause unacceptable damage by absorbing energy, expanding and causing small craters on the glass surface. A cerium oxide particle at various depths was modeled to investigate the phenomenology of crater formation. There was a strong surface interaction that 
influenced the formation and direction of the damage (cracking) that ultimately resulted in the blow-out of the damaged material leaving a relatively clean crater in the glass. The depth of the contaminant played an important role in determining the crater dimensions. A $100 \mathrm{~nm}$ diameter contaminant, placed at a depth of $150 \mathrm{~nm}$ and $300 \mathrm{~nm}$, resulted in crater surface diameters of $600 \mathrm{~nm}$ and $1300 \mathrm{~nm}$ respectively. 


\subsection{References:}

1 J. Wackerle, Shock-Wave Compression of Quartz, J. Appl. Phys., 33, p.922 (1962)

2 H. Sugiura, K. Kondo, and A. Sawaoka, Dynamic Response of Fused Quartz in the Permanent Densification Region, J. Appl. Phys., 52, p.3375 (1981)

3 D.E. Grady, and R.E. Hollenbach, Dynamic Fracture Strength of Rock, Geoph Res. Lt, 6, p.73 (1979)

4 Z. Rosenberg, On the Relation Between the Hugoniot Elastic Limit and the Yield Strength of Brittle Material, J. Appl. Phys., 74, p.752 (1993)

5 G.I. Kanel, S.V. Rasorenov, and V.E. Fortov, The Failure Waves and Spallation in Homogeneous Brittle Materials, Shock Compression of Condensed Matter, p.450 (1991)

6 N.S. Brar, and S.J. Bless, Impact-Induced Failure Waves in Glass Bars and Plates, Appl. Phys. Lett., 59, p.3396 (1991)

7 N.K. Bourne, Z. Rosenberg, and J.E. Field, High-Speed Photography of Compressive Failure Waves in Glasses, J. Appl. Phys., 78, p.3736 (1995)

8 D.R. Faux, Brittle Damage Models in DYNA2D, LLNL, UCRL-ID-128520, September, 1997

9 F.R. Tuler, and B.M. Butcher, A Criterion for the Time Dependence of Dynamic Fracture, Int. J. Fracture Mechanics, Vol. 4, No. 4, December 1968.

10 M. Boustie, F. Cottet, Y. Chauveau, Spalling of Aluminum and Copper Targets by Laser Shocks, Shock Waves and High Strain Rate Phenomena in Materials, Edited by M.A. Meyer, L.E. Murr, K.P. Staudhammer, p.741

11 D.J. Steinberg, Equation of State and Strength Properties of Selected Materials, UCRL-MA-106439, 1996

12 A.M. Rubenchik, LLNL, private communication, 1996

13 M.D. Feit, LLNL, private communication, 1997

14 B. Penetrante, LLNL, private communication, 1997

15 F. Genin and L. Yoshiyama, A Study of Surface Damage Morphologies in Superpolished Fused Silica at 355 nm, LLNL Internal Memo NIF-0001984, 1997 


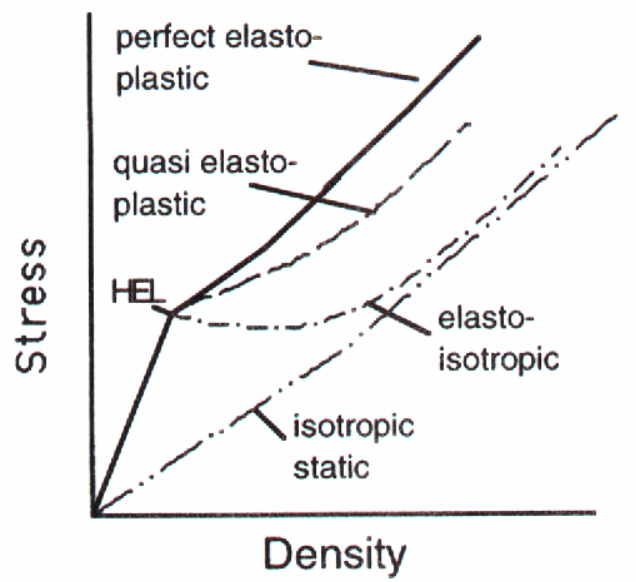

Figure 1: Typical Compression Curves.

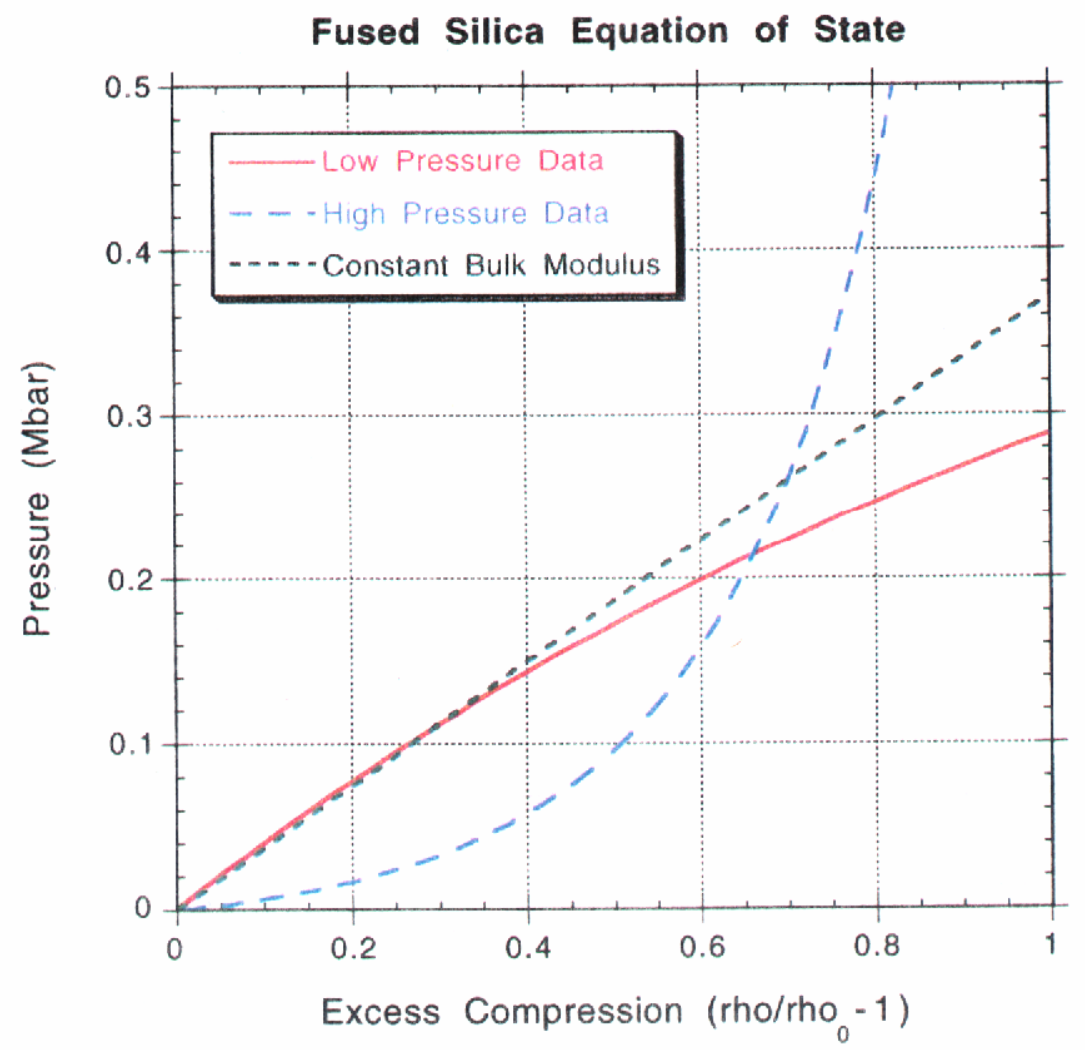

Figure 2: Equation of State Response for Fused Silica. 


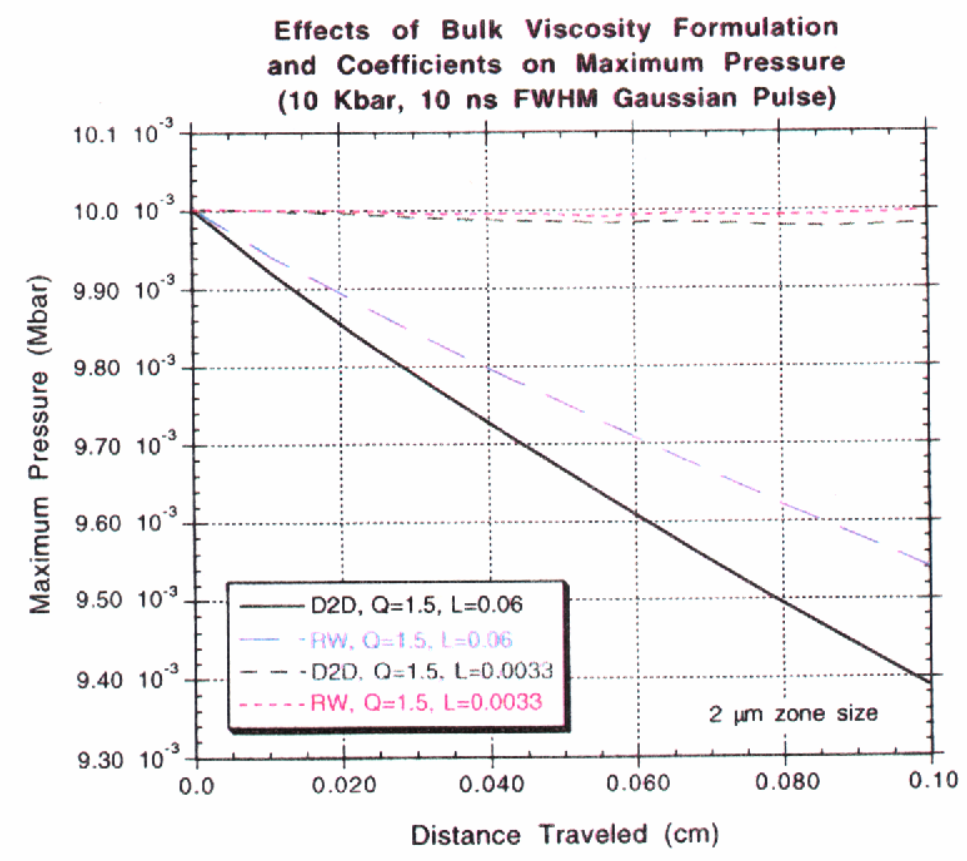

Figure 3: One-Dimensional Plane Strain Analysis of Pressure Pulse Traveling Down Bar. Effect of Artificial Viscosity Method and Coefficients on Pressure Pulse.

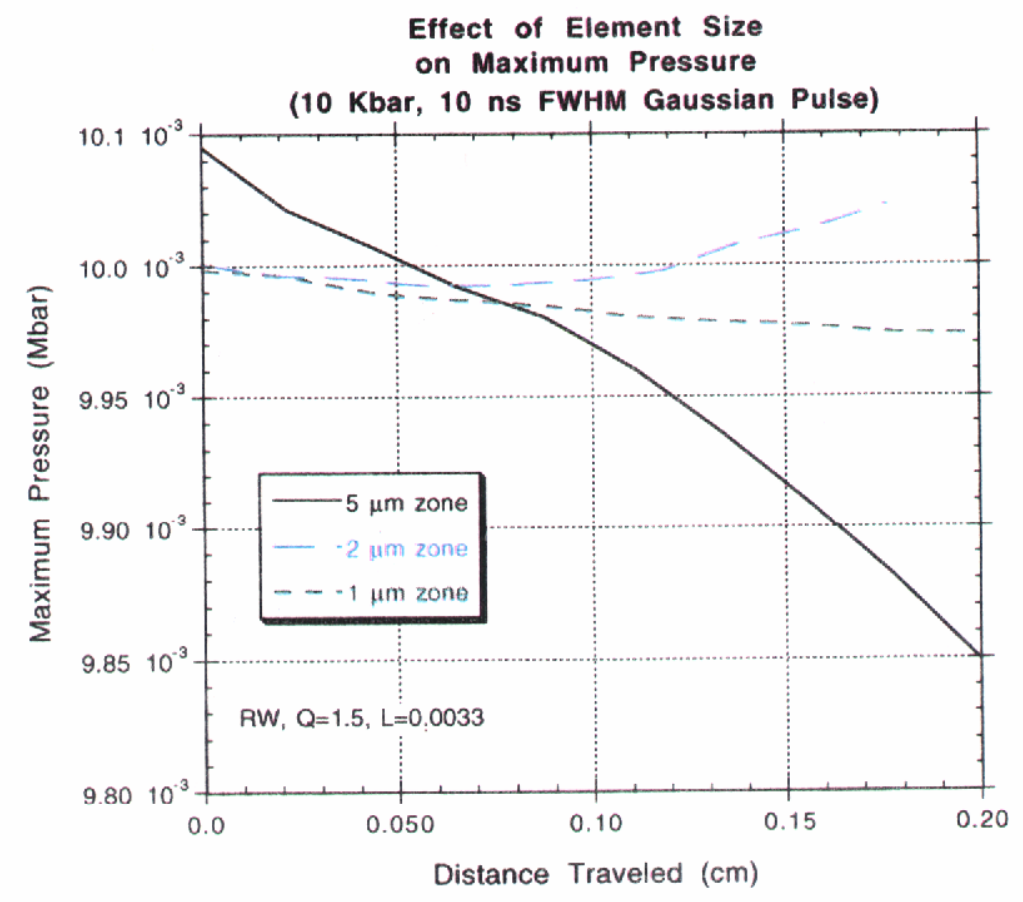

Figure 4: One-Dimensional Plane Strain Analysis of Pressure Pulse Traveling Down Bar. Effect of Element Size on Pressure Pulse. 


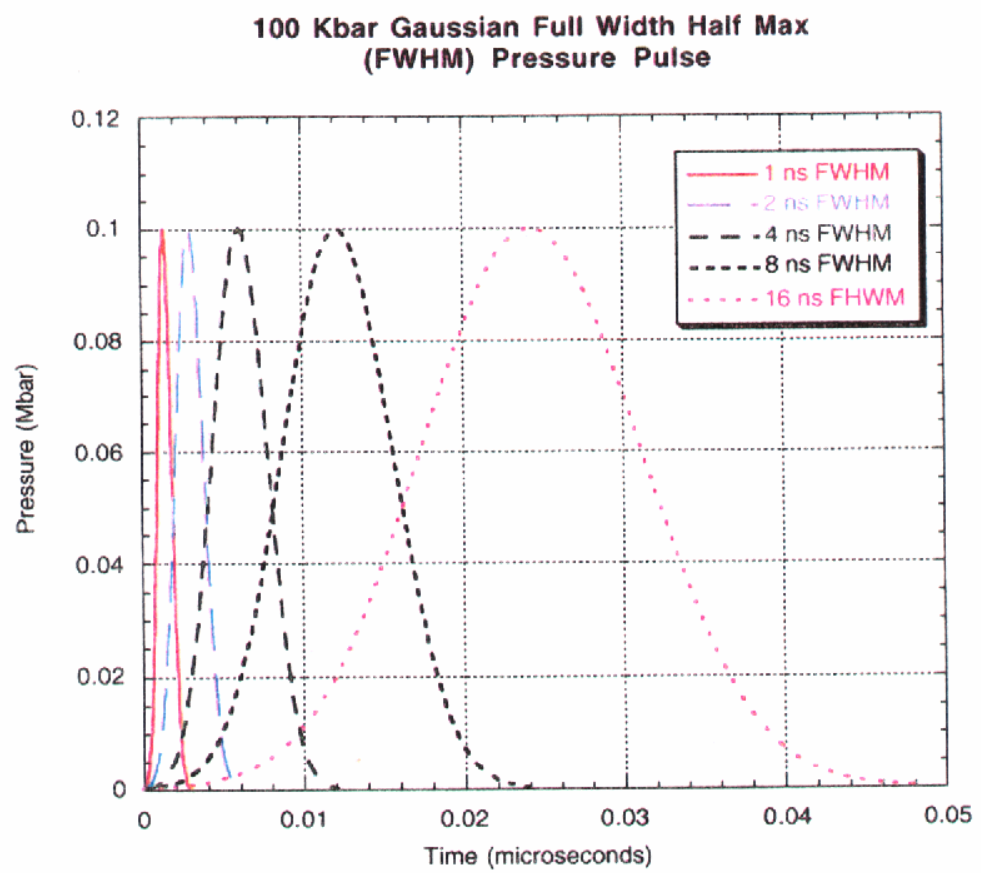

Figure 5: 100 Kbars Gaussian Pressure Pulses Used in One-Dimensional Spall Calculations.

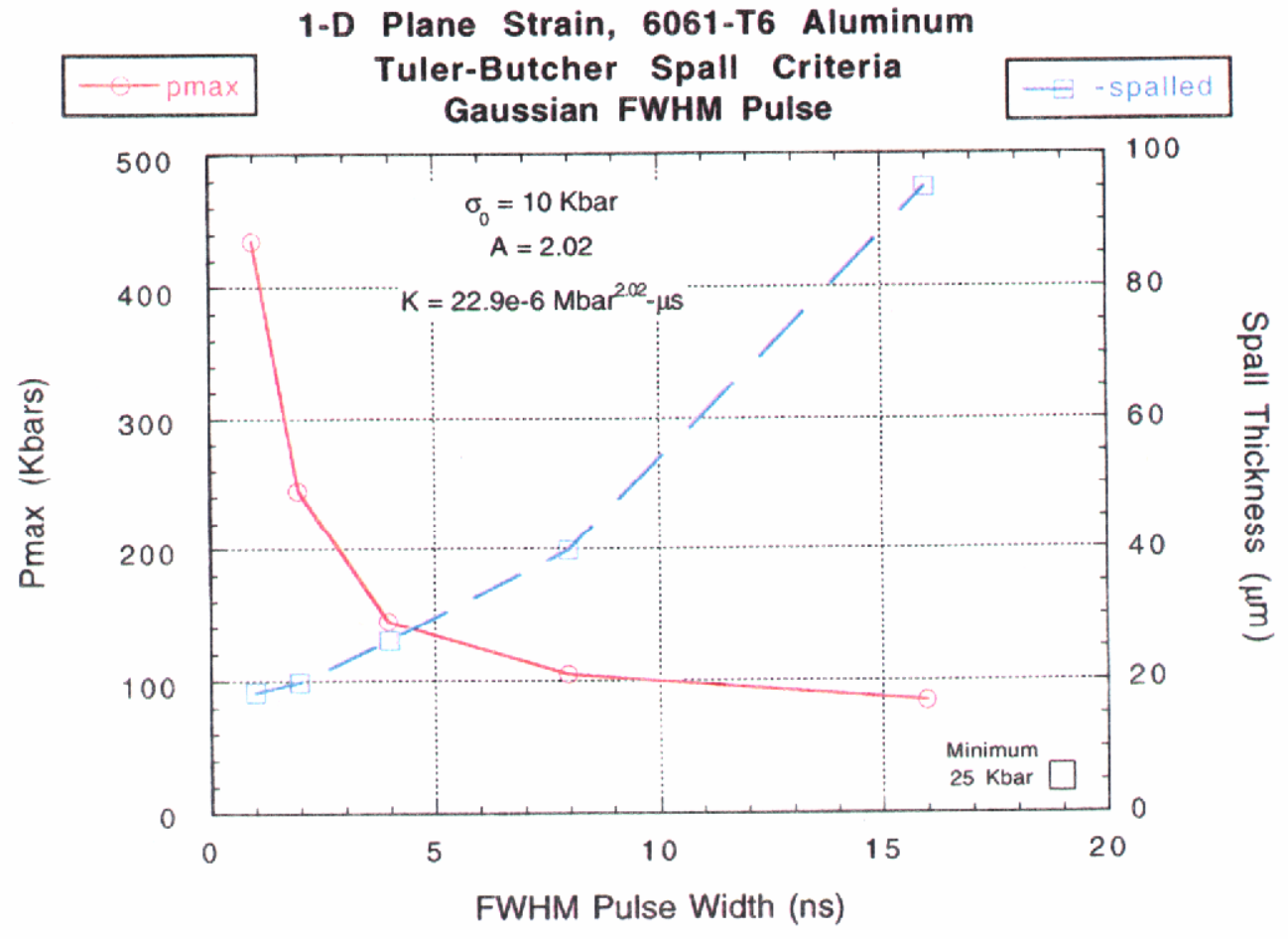

Figure 6: Minimum Pressure Required to Cause Spallation for a Given Pressure Pulse and Spallation Thickness. 


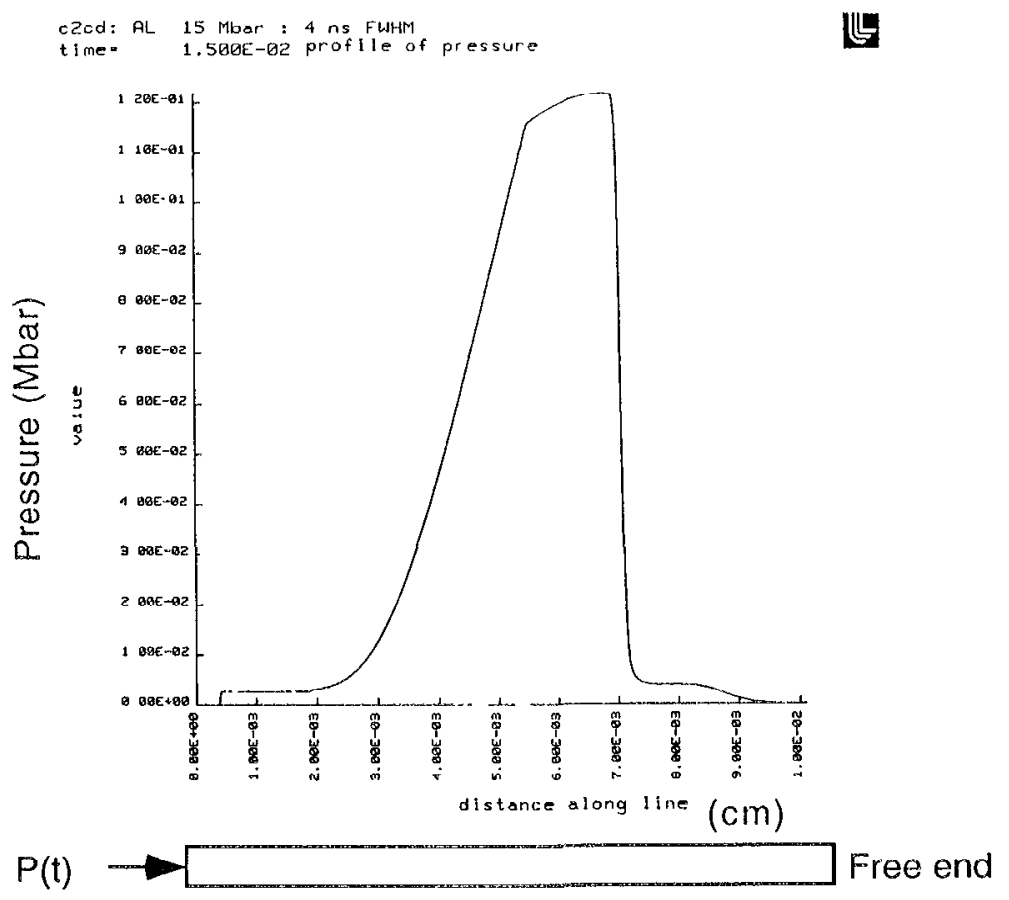

Figure 7: Line Drawing of Pressure vs. Distance Along Aluminum Bar. Pressure Wave Travels Left to Right.

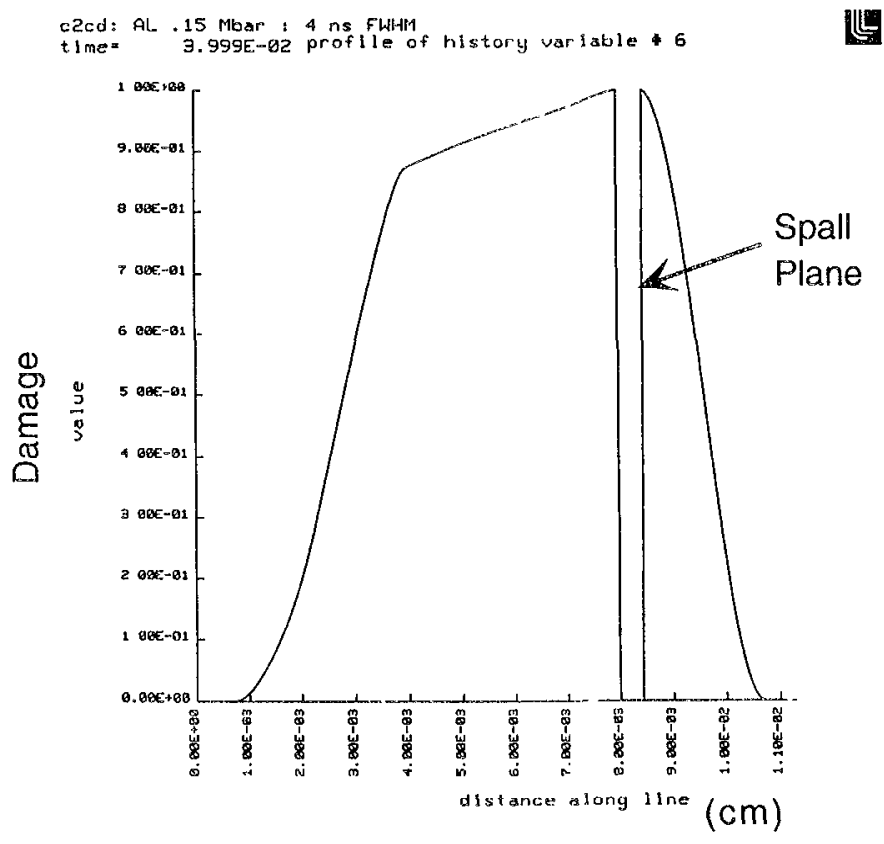

Figure 8: Line Drawing of Material Damage vs. Distance Along Aluminum Bar. Damage Greater Than One Means Failure. 


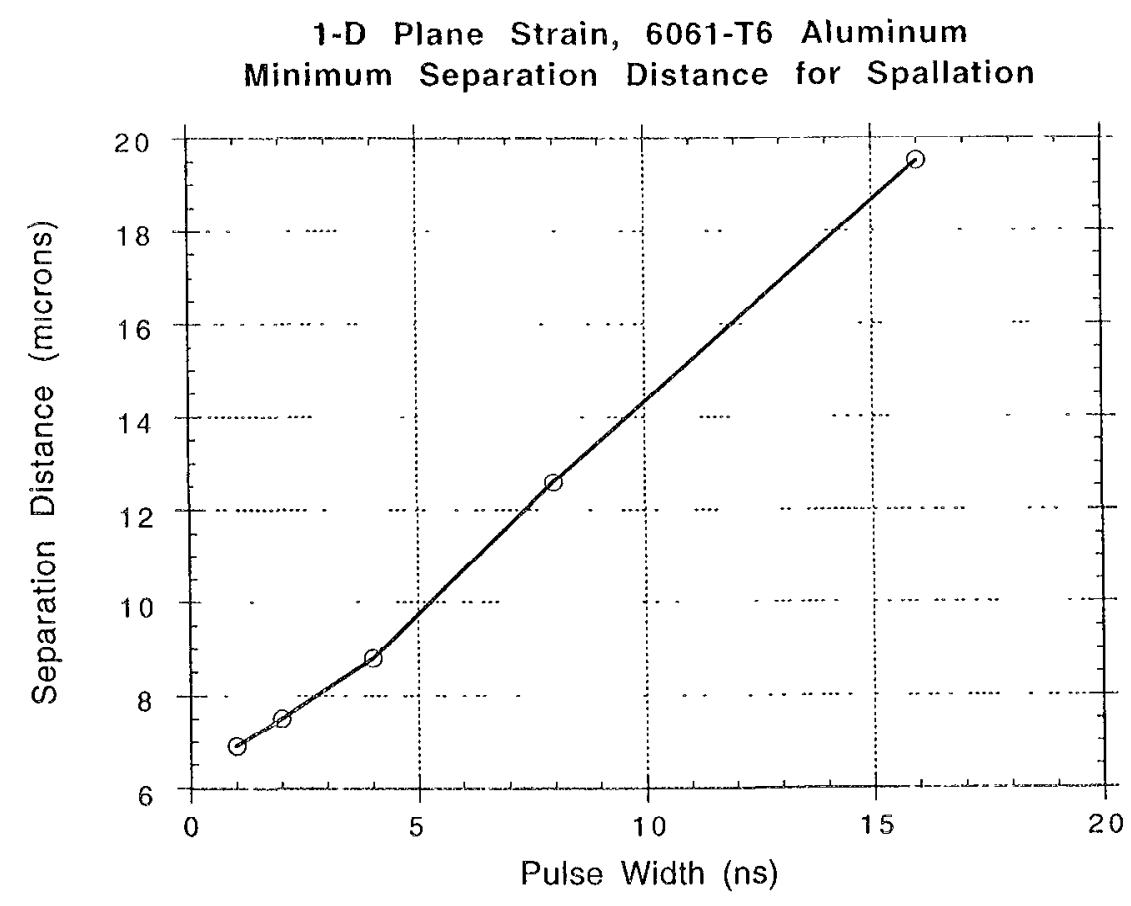

Figure 9: Minimum Distance the Surface Particle Must Be from the Optic for Spallation to Occur for Various Pulse Widths.

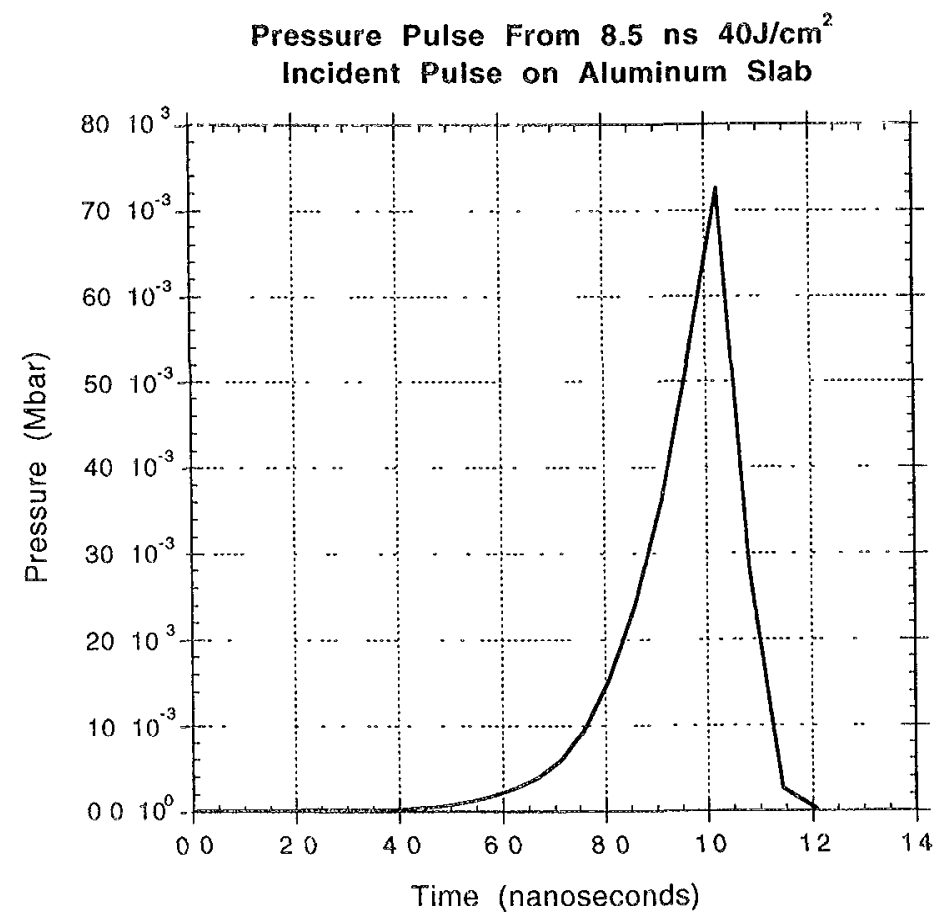

Figure 10: Pressure Pulse from an $8.5 \mathrm{~ns} 40 \mathrm{~J} / \mathrm{cm}^{2}$ Incident Pulse on an Aluminum Slab. 


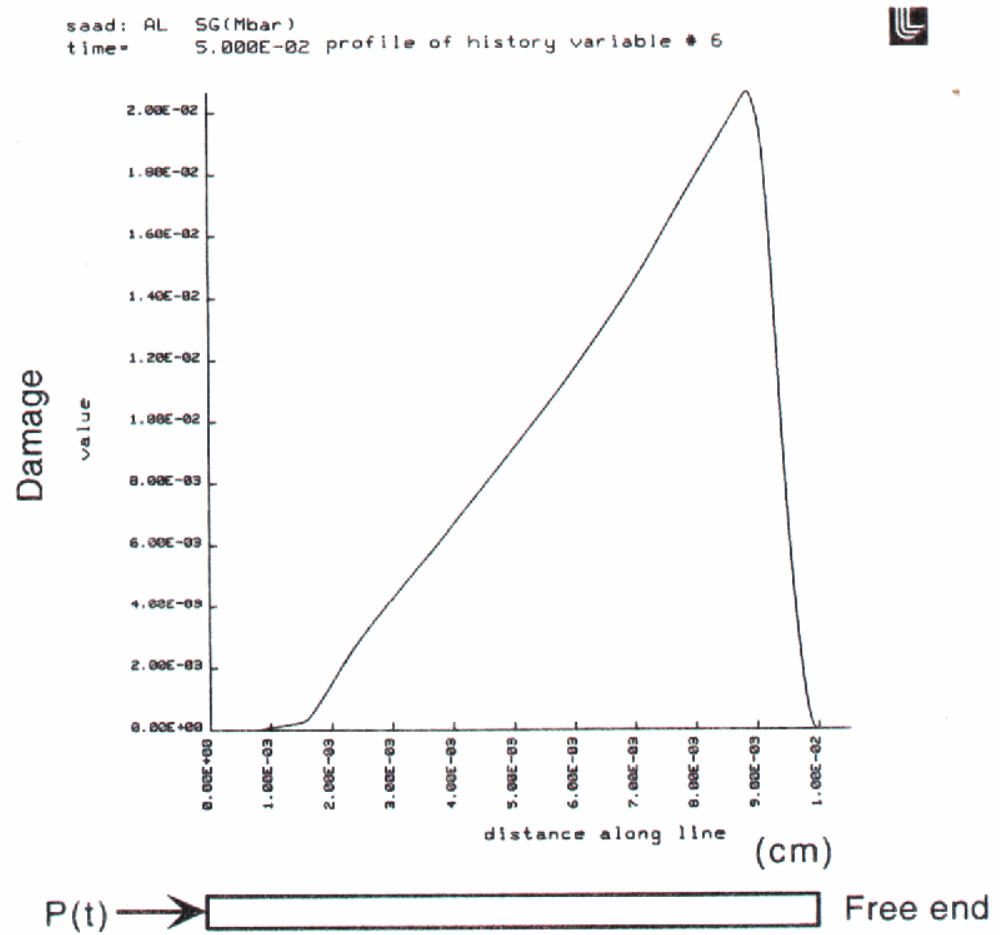

Figure 11: Line Drawing of Material Damage vs. Distance Along Aluminum Bar. Damage Greater than One means Failure.

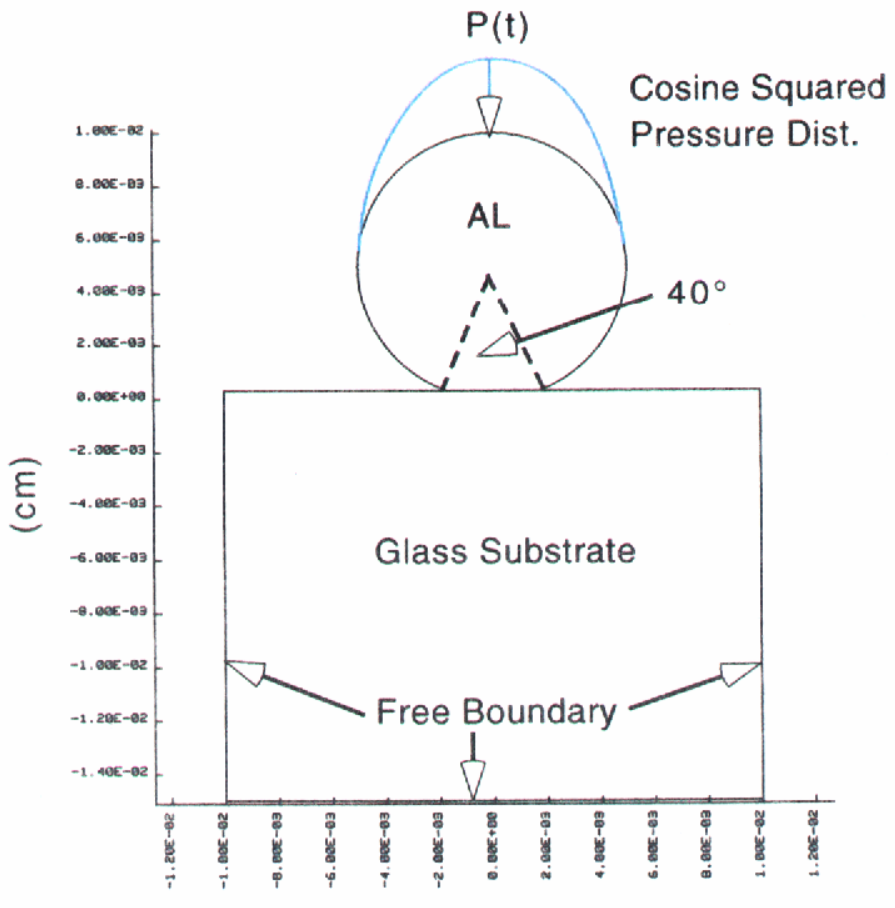

$(\mathrm{cm})$

Figure 12: Aluminum Sphere on Surface of Optic with a Cosine Squared Pressure Distribution. $40^{\circ}$ Contact. 

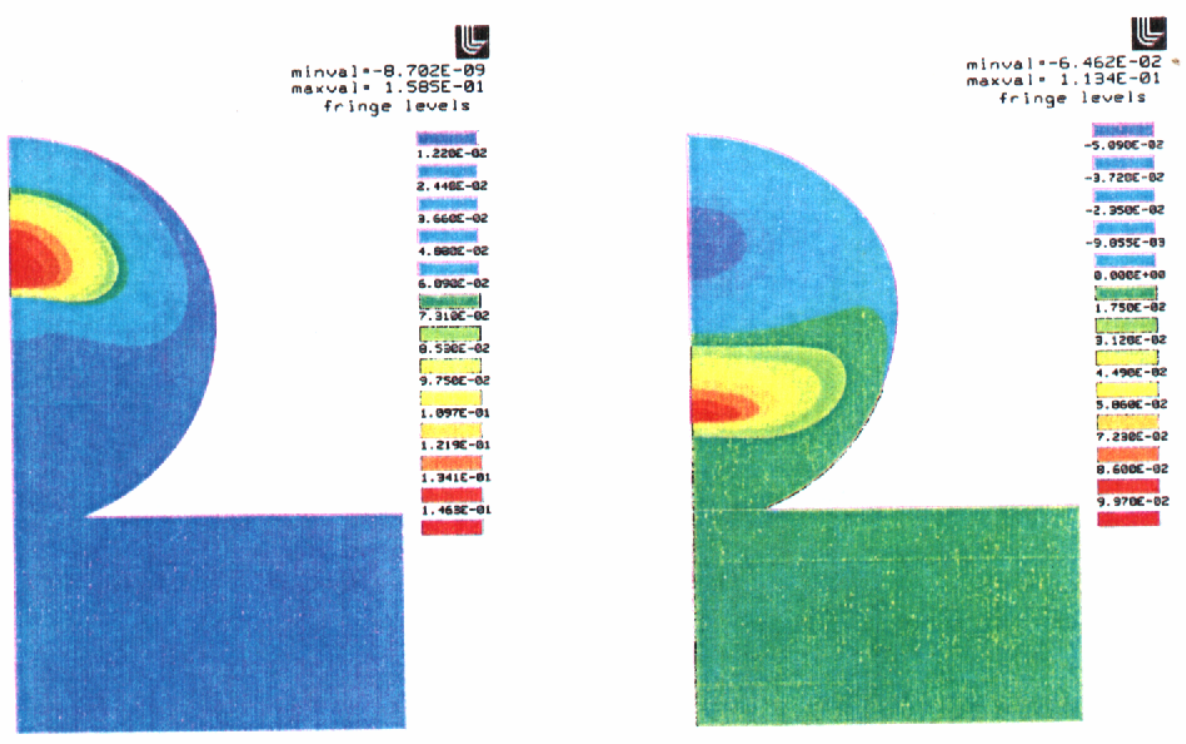

Figure 13: 100 Microns Diameter, $40^{\circ}$ Contact Angle, Aluminum Sphere; 150 Kbars 4 ns FWHM Gaussian Pulse; Fringe of Pressure (Mbars) in Aluminum Particle. a) Time $=10$ nanoseconds, $b$ ) Time $=15$ nanoseconds.
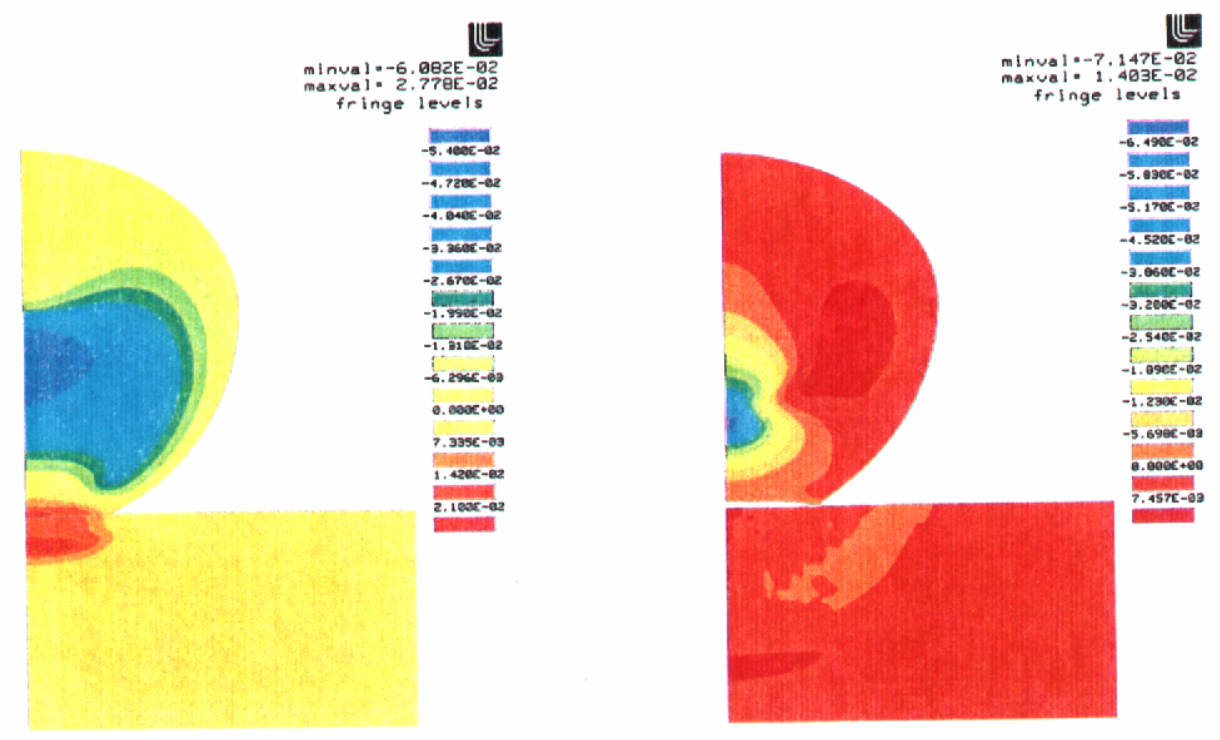

Figure 14: 100 Microns Diameter, $40^{\circ}$ Contact Angle, Aluminum Sphere; 150 Kbars 4 ns FWHM Gaussian Pulse; Fringe of Pressure (Mbars) in Aluminum Particle. a) Time $=20$ nanoseconds, $b$ ) Time $=25$ nanoseconds. 


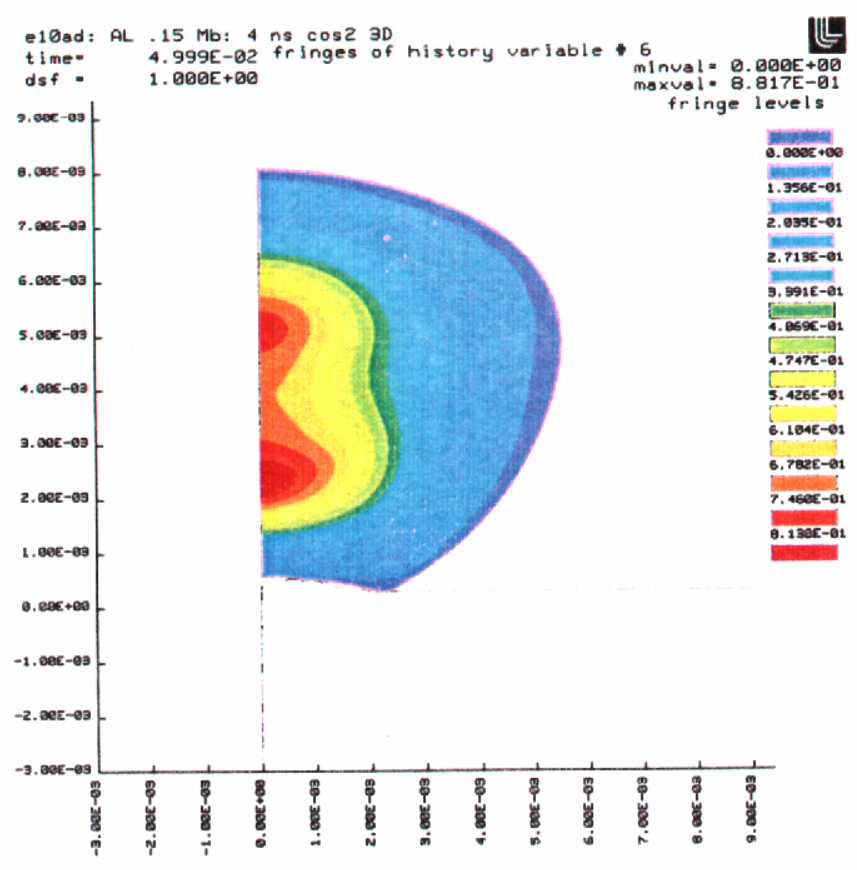

Figure 15: 100 Microns Diameter, $40^{\circ}$ Contact Angle, Aluminum Sphere; 150 Kbars 4 ns FWHM Gaussian Pulse; Time $=50$ nanoseconds. Fringe of Damage in Aluminum Particle.

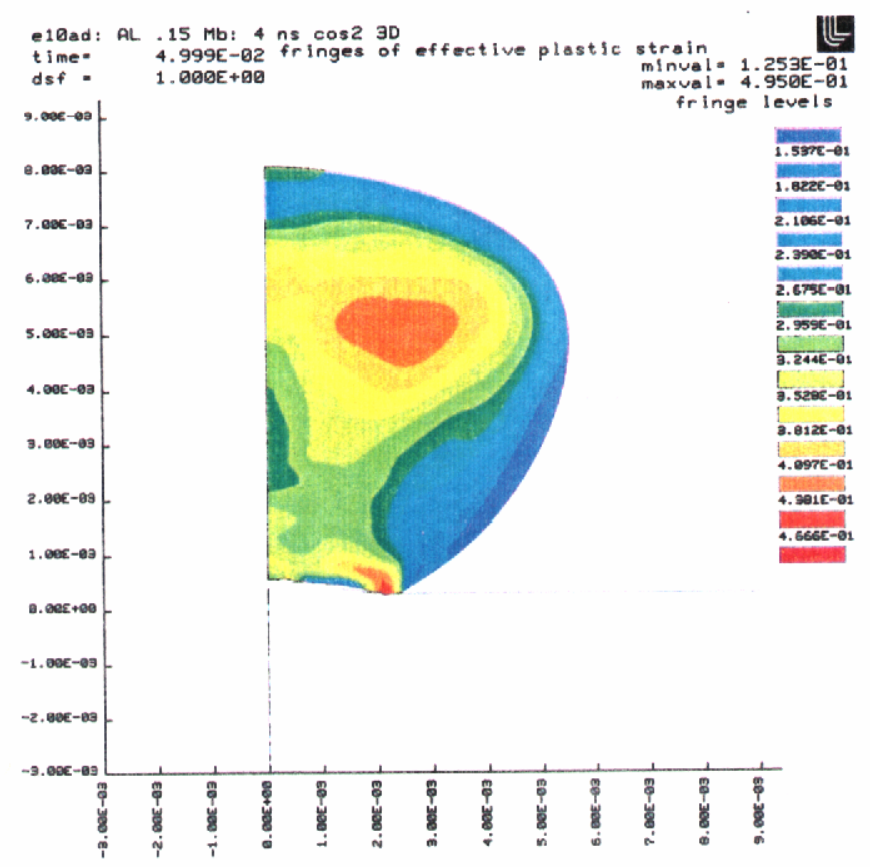

Figure 16: 100 Microns Diameter, $40^{\circ}$ Contact Angle, Aluminum Sphere; 150 Kbars 4 ns FWHM Gaussian Pulse; Time $=50$ nanoseconds. Fringe of Effective Plastic Strain in Aluminum Particle. 


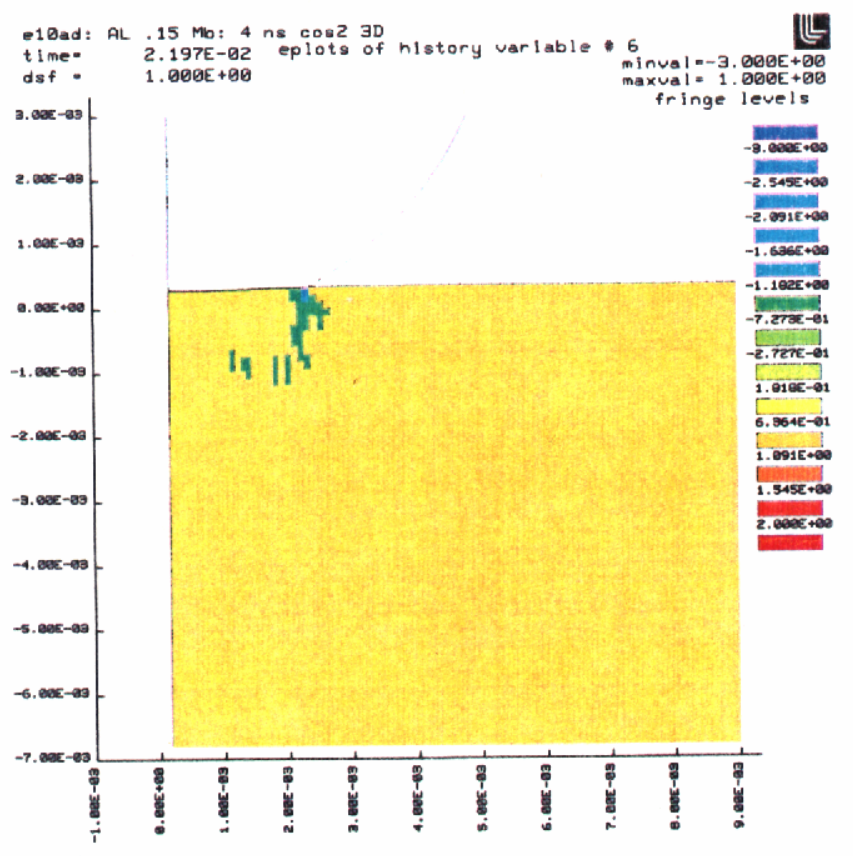

Figure 17: 100 Microns Diameter, $40^{\circ}$ Contact Angle, Aluminum Sphere; 150 Kbars 4 ns FWHM Gaussian Pulse; Time $=22$ nanoseconds. Fringe of Damage in Glass Substrate.

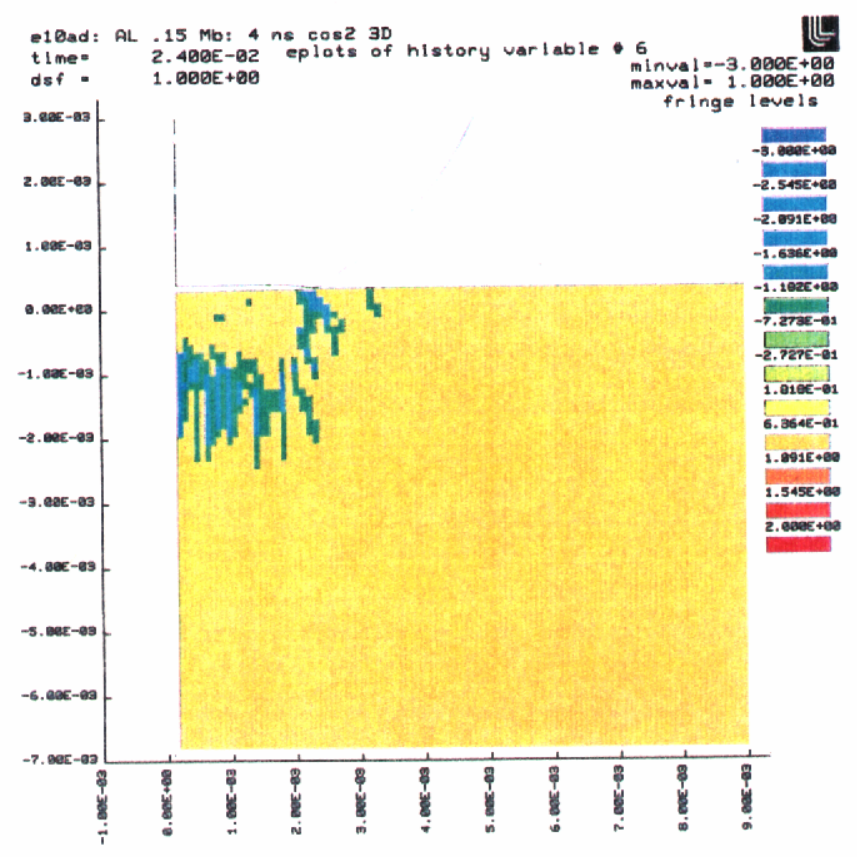

Figure 18: 100 Microns Diameter, $40^{\circ}$ Contact Angle, Aluminum Sphere; 150 Kbars 4 ns FWHM Gaussian Pulse; Time $=24$ nanoseconds. Fringe of Damage in Glass Substrate. 


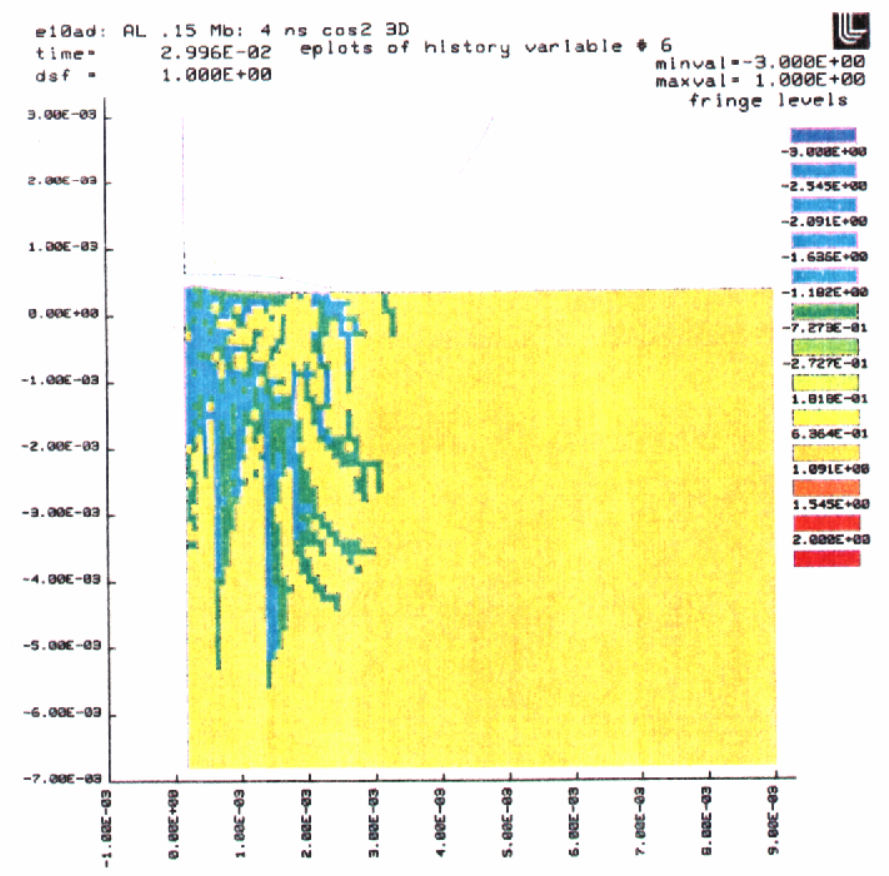

Figure 19: 100 Microns Diameter, $40^{\circ}$ Contact Angle, Aluminum Sphere; 150 Kbars 4 ns FWHM Gaussian Pulse; Time $=30$ nanoseconds. Fringe of Damage in Glass Substrate.

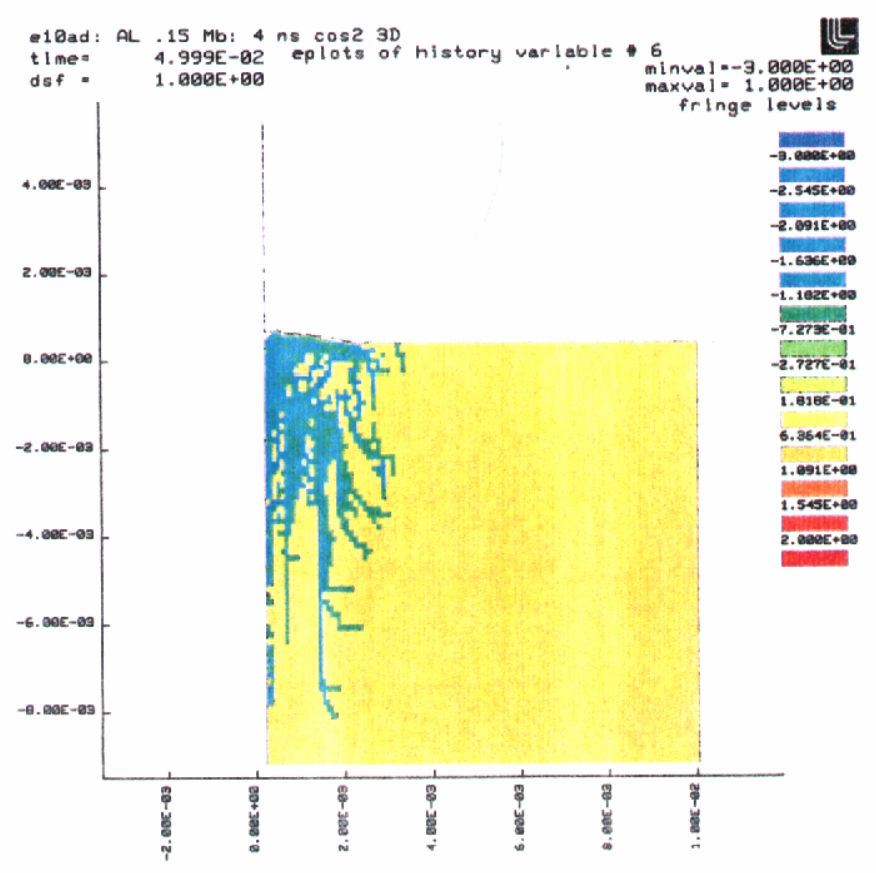

Figure 20: 100 Microns Diameter, $40^{\circ}$ Contact Angle, Aluminum Sphere; 150 Kbars 4 ns FWHM Gaussian Pulse; Time $=50$ nanoseconds. Fringe of Damage in Glass Substrate. 
UCRL-ID-1 28908

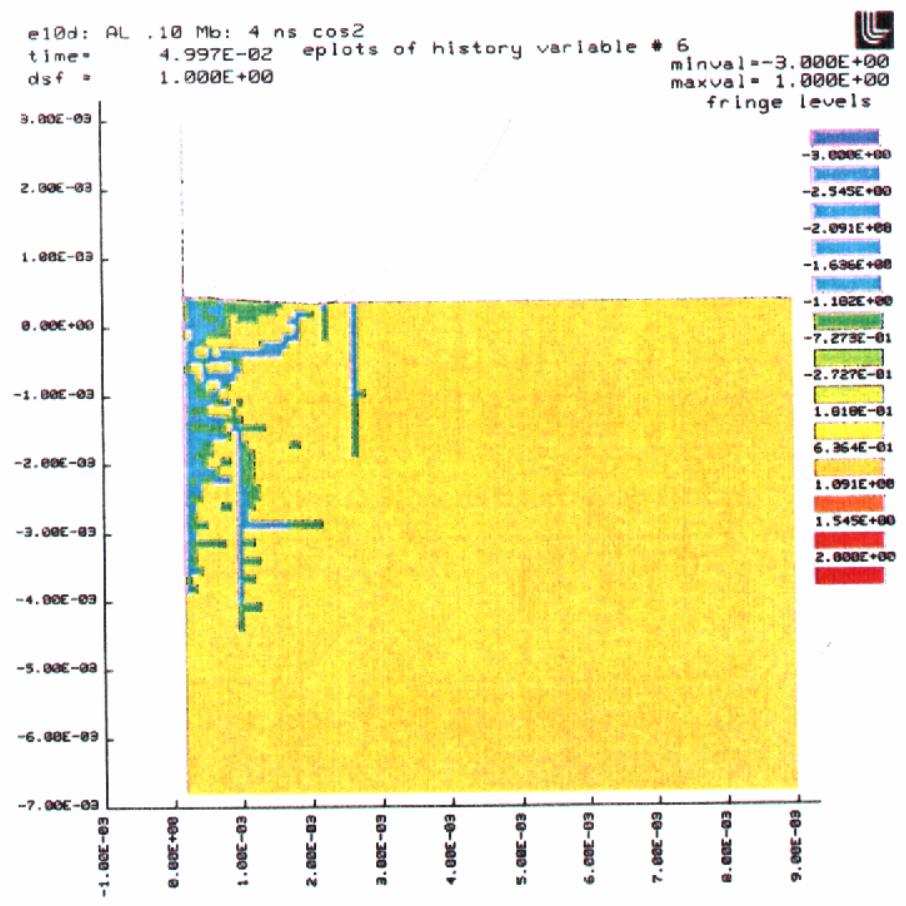

Figure 21: 100 Microns Diameter, $40^{\circ}$ Contact Angle, Aluminum Sphere; 100 Kbars 4 ns FWHM Gaussian Pulse; Time $=50$ nanoseconds; Fringe of Damage in Glass Substrate.

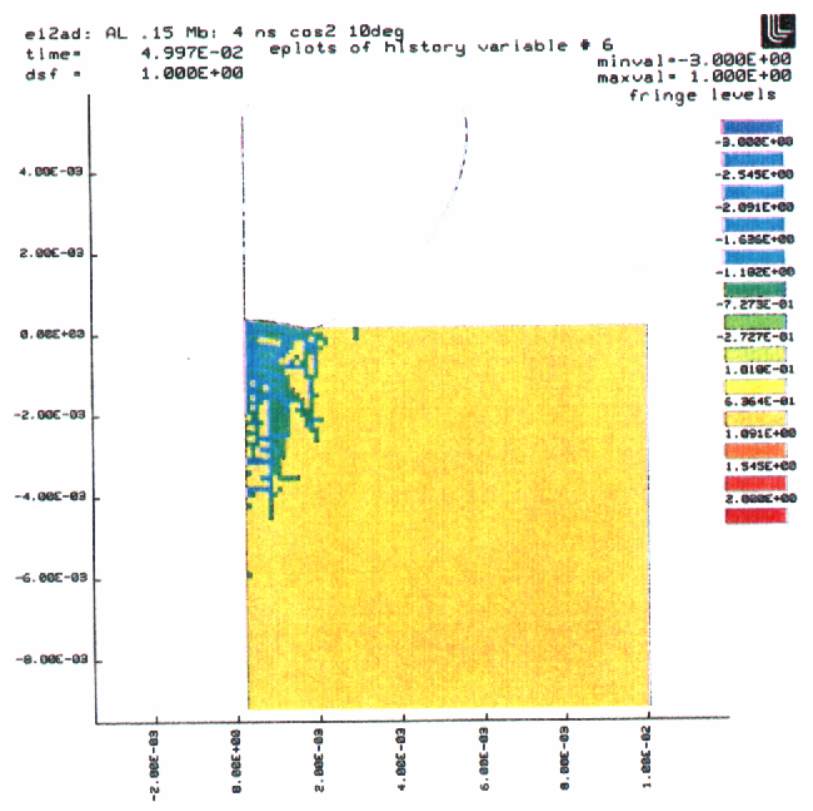

Figure 22: 100 Micron Diameter, $20^{\circ}$ Contact Angle, Aluminum Sphere; 150 Kbars $4 \mathrm{~ns}$ FWHM Gaussian Pulse; Time $=50$ nonoseconds. Fringe of Damage in Glass Substrate. 


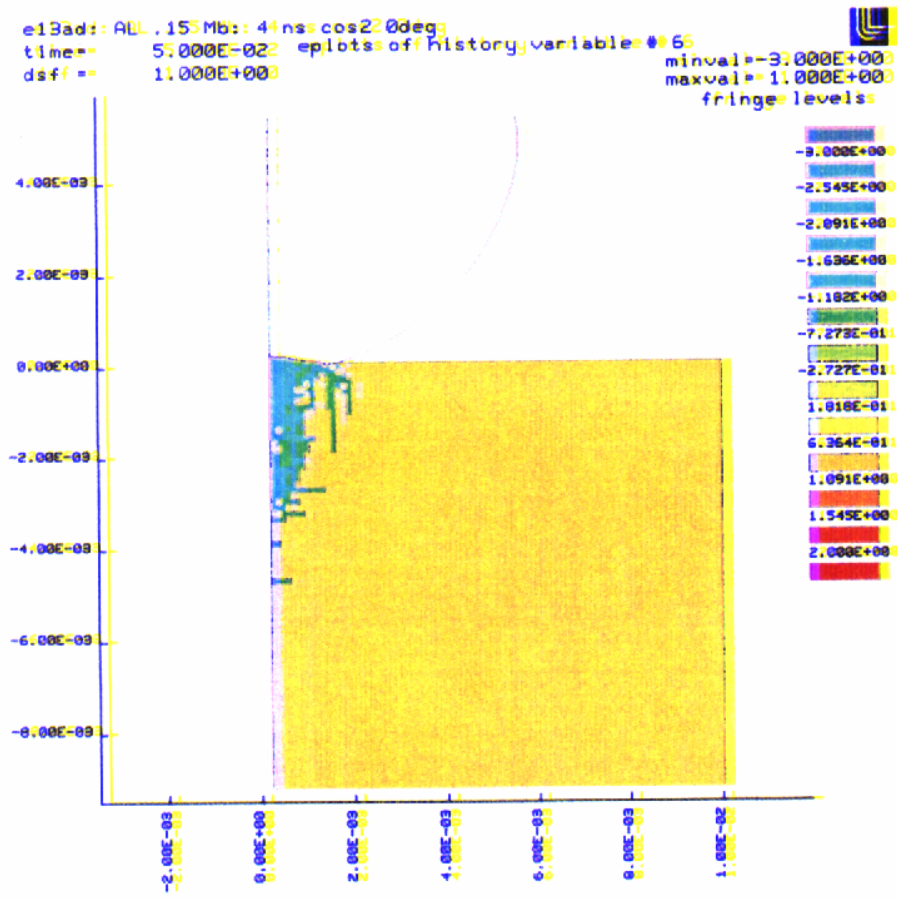

Figure 23: 100 Microns Diameter, $0^{\circ}$ Contact Angle, Aluminum Sphere; 150 Kbars 4 ns FWHM Gaussian Pulse; Time $=50$ nonoseconds. Fringe of Damage in Glass Substrate.

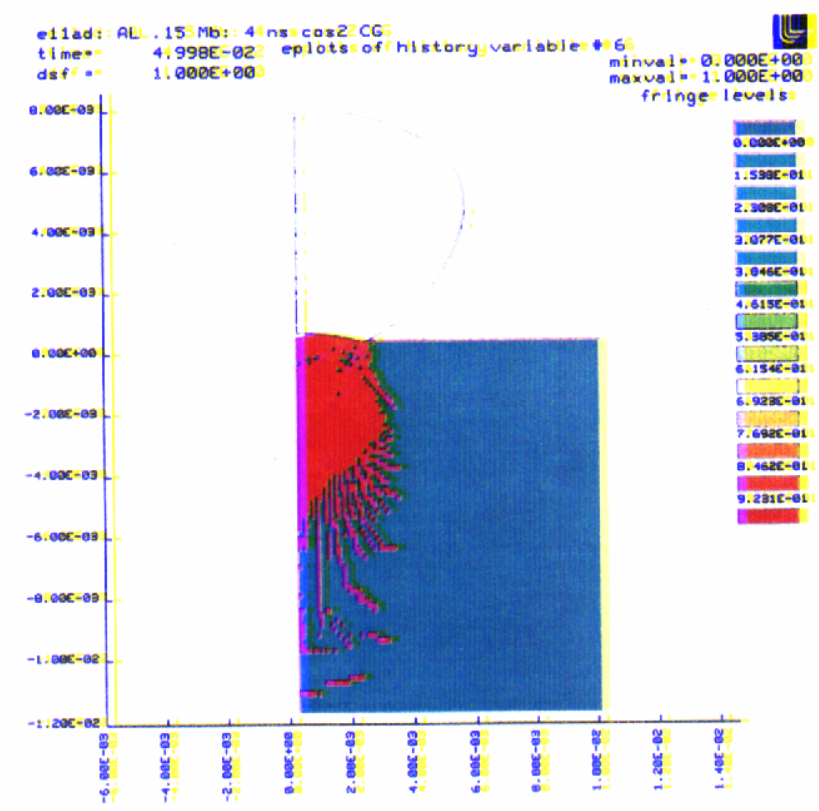

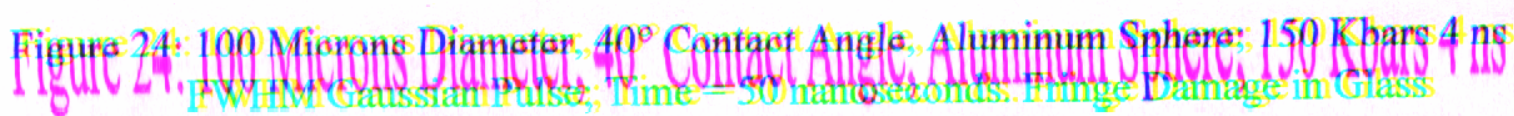

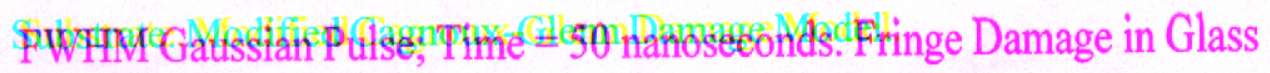
Substrate. Modified Cagnoux-Glenn Damage Model. 


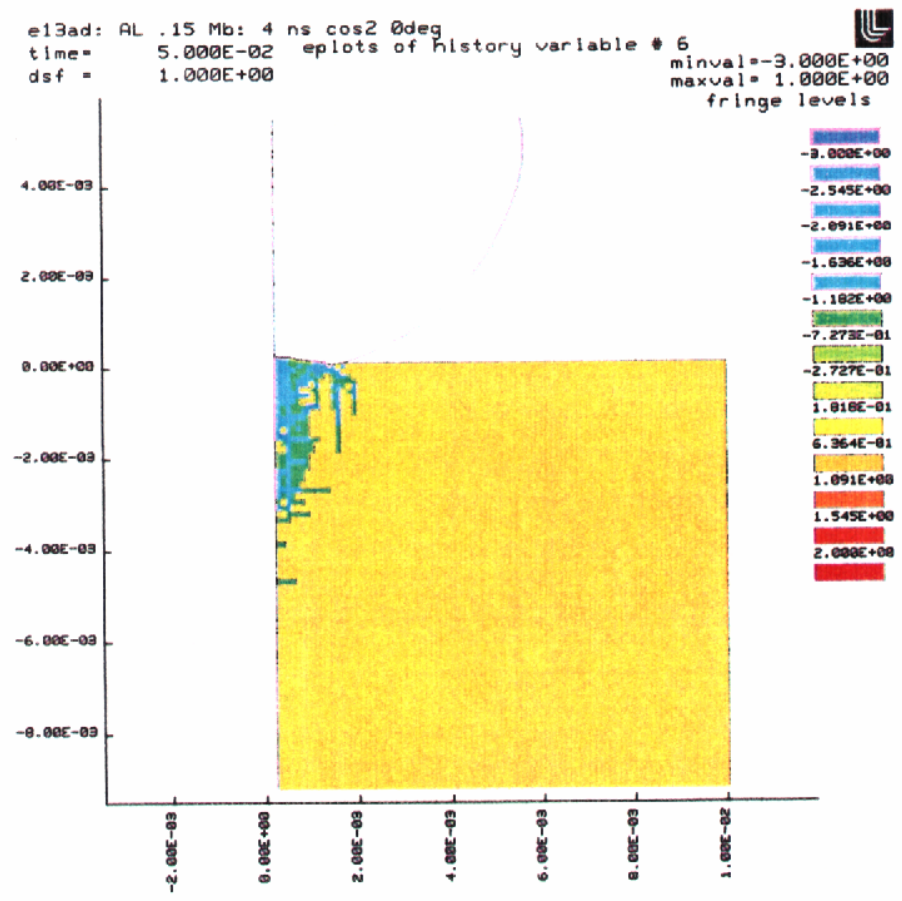

Figure 23: 100 Microns Diameter, $0^{\circ}$ Contact Angle, Aluminum Sphere; 150 Kbars 4 ns FWHM Gaussian Pulse; Time $=50$ nonoseconds. Fringe of Damage in Glass Substrate.

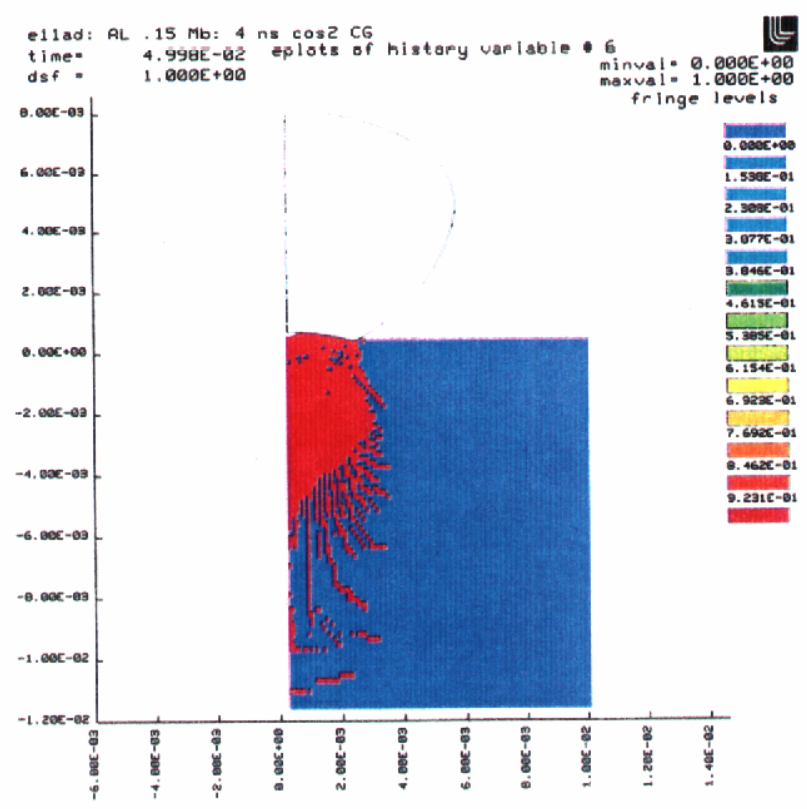

Figure 24: 100 Microns Diameter, $40^{\circ}$ Contact Angle, Aluminum Sphere; 150 Kbars 4 ns FWHM Gaussian Pulse; Time $=50$ nanoseconds. Fringe Damage in Glass Substrate. Modified Cagnoux-Glenn Damage Model. 


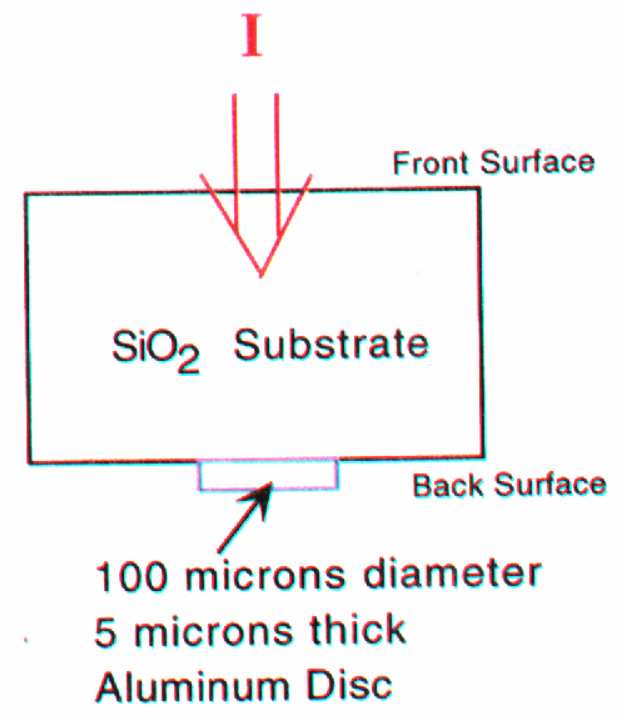

Figure 25: 100 Microns Diameter, 5 Microns Thick Aluminum Disc on $\mathrm{SiO}_{2}$ Back Surface; $18 \mathrm{~J} / \mathrm{cm}^{3}$ Gaussian Pulse, 3 ns FWHM.

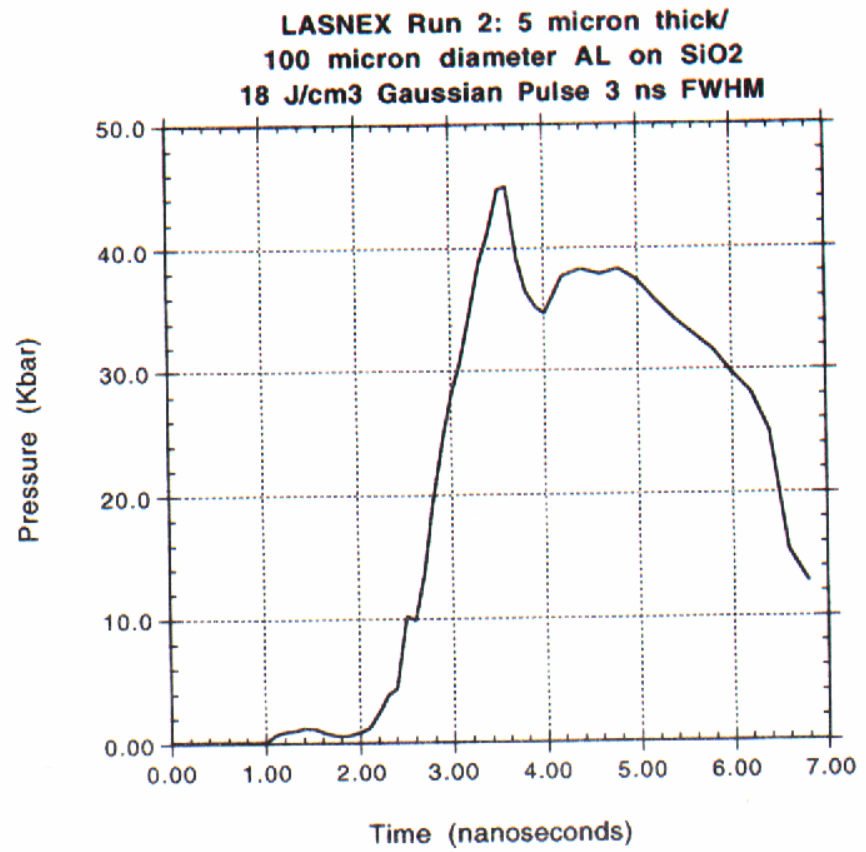

Figure 26: 100 Microns Diameter, 5 Microns Thick Aluminum Disc on $\mathrm{SiO}_{2}$ Back Surface; $18 \mathrm{~J} / \mathrm{cm}^{3}$ Gaussian Pulse, $3 \mathrm{~ns}$ FWHM. Pressure History at Radius = 0 microns. 
UCRL-ID-128908

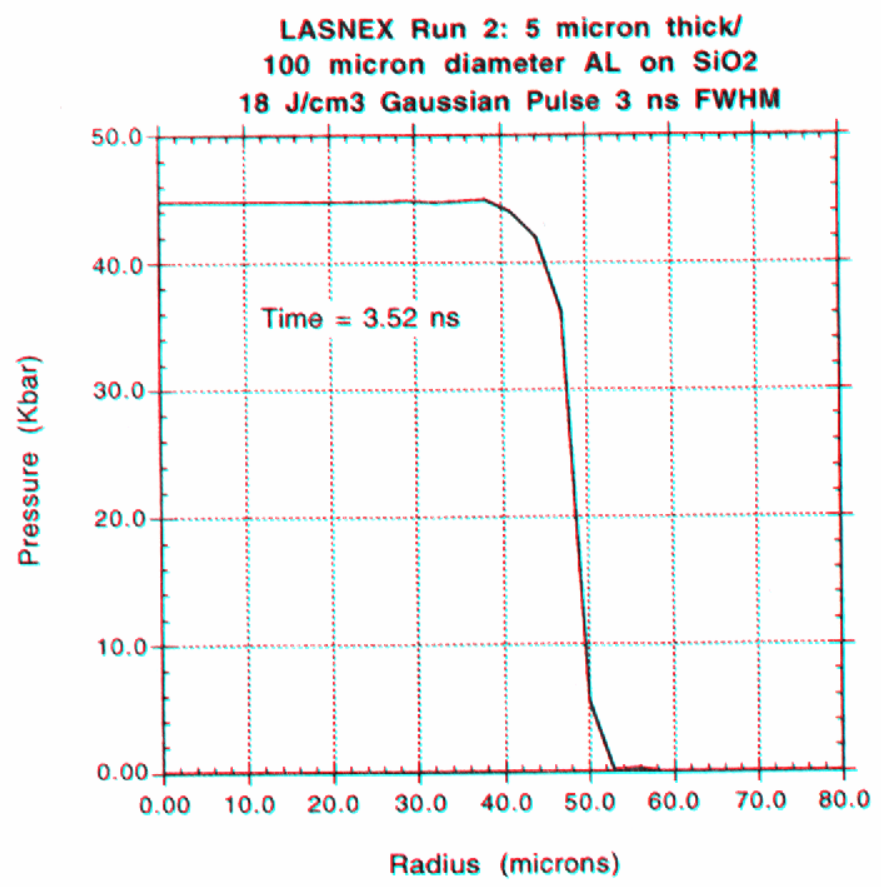

Figure 27: 100 Microns Diameter, 5 Microns Thick Aluminum Disc on $\mathrm{SiO}_{2}$ Back

Surface; $18 \mathrm{~J} / \mathrm{cm}^{3}$ Gaussian Pulse, $3 \mathrm{~ns}$ FWHM. Pressure vs. Radius at Time $=$ $3.52 \mathrm{~ns}$.

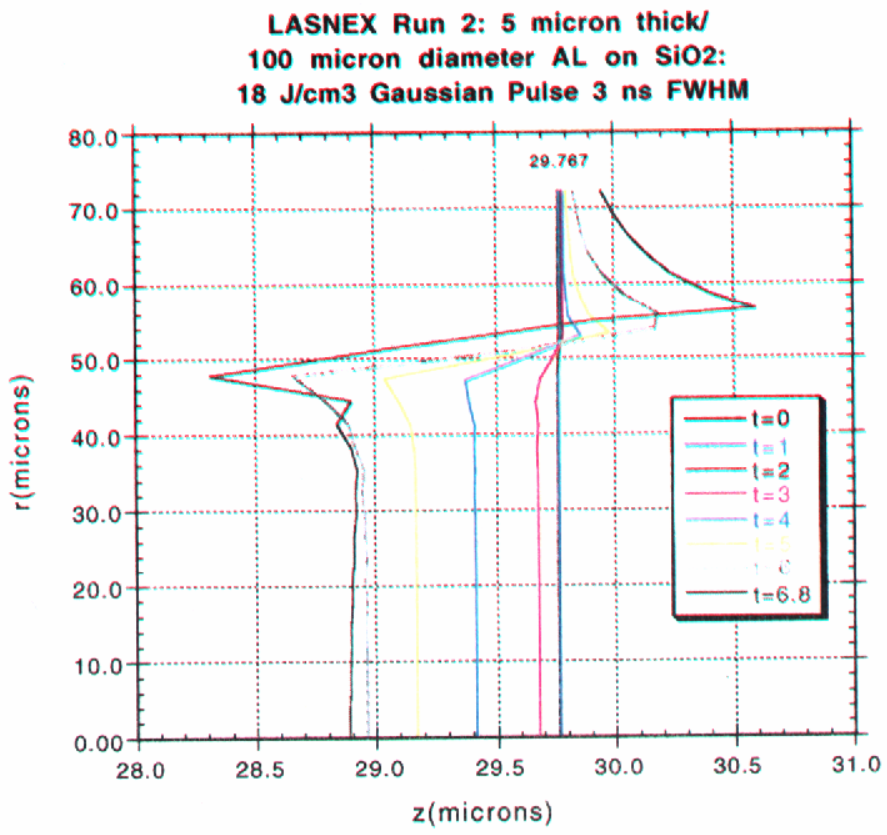

Figure 28: 100 Microns Diameter, 5 Microns Thick Aluminum Disc on $\mathrm{SiO}_{2}$ Back Surface; $18 \mathrm{~J} / \mathrm{cm}^{3}$ Gaussian Pulse, $3 \mathrm{~ns}$ FWHM. Interface Between Solid and Melt $(0.1 \mathrm{eV})$ at Various Times Where Pressure Is Recorded. 


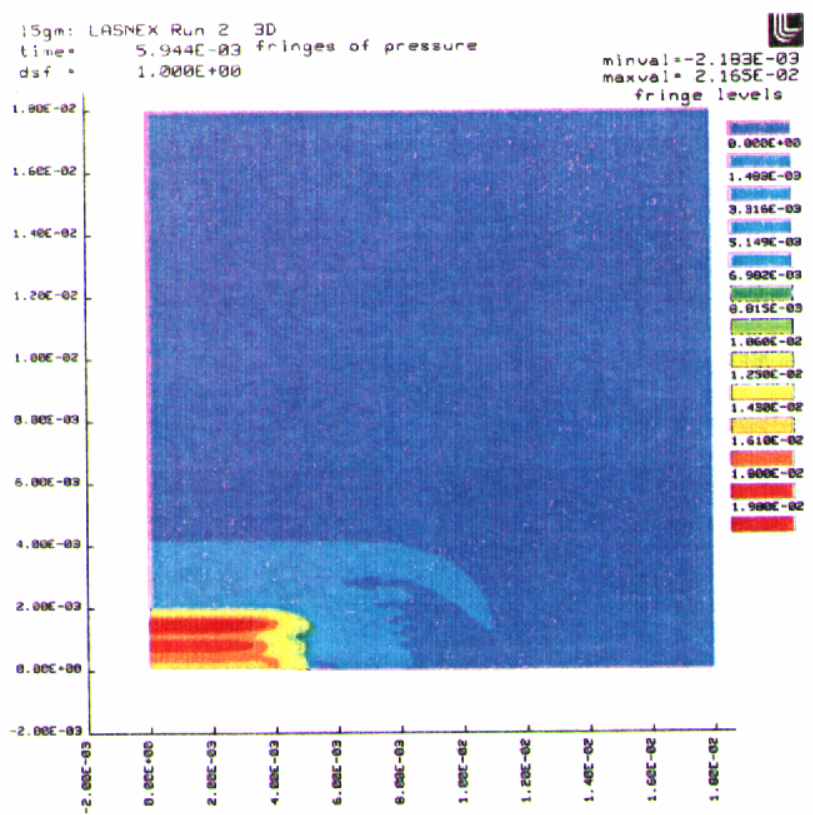

Figure 29: 100 Microns Diameter, 5 Microns Thick Aluminum Disc on $\mathrm{SiO}_{2}$ Back Surface; $18 \mathrm{~J} / \mathrm{cm}^{3}$ Gaussian Pulse, $3 \mathrm{~ns}$ FWHM. Fringe of Pressure (Mbars), Time $=6 \mathrm{~ns}$.

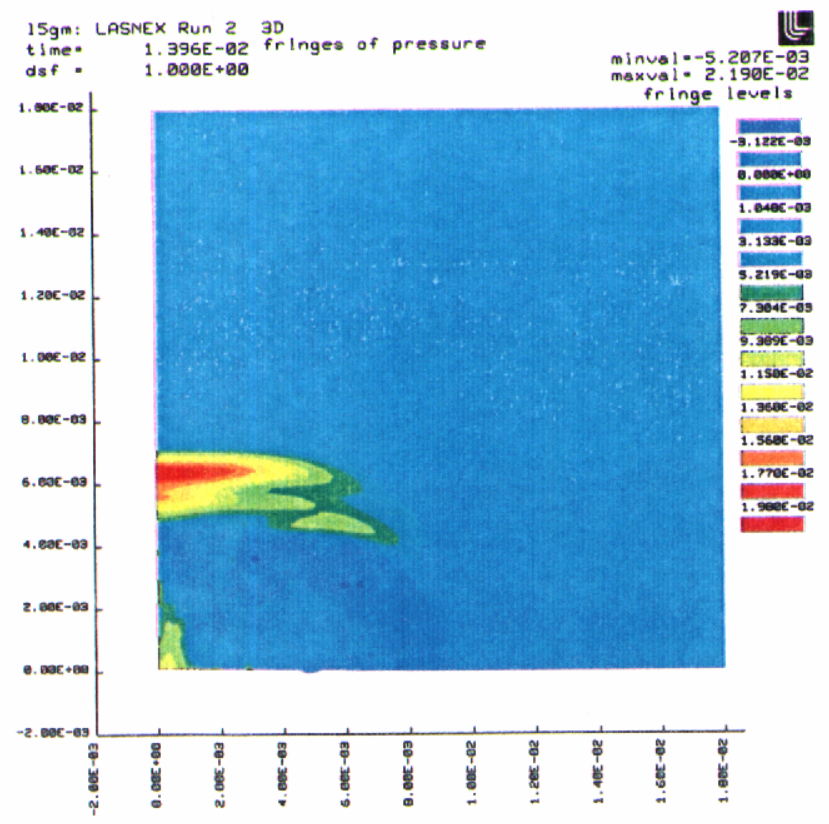

Figure 30: 100 Microns Diameter, 5 Microns Thick Aluminum Disc on $\mathrm{SiO}_{2}$ Back Surface; $18 \mathrm{~J} / \mathrm{cm}^{3}$ Gaussian Pulse, $3 \mathrm{~ns}$ FWHM. Fringe of Pressure (Mbars), Time $=14 \mathrm{~ns}$. 
UCRL-ID-1 28908

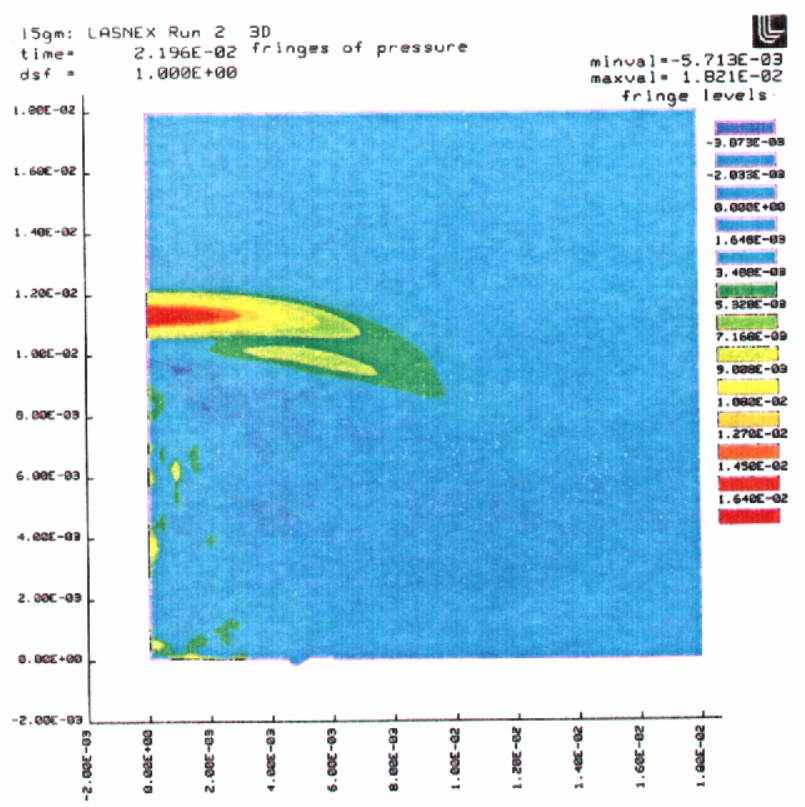

Figure 31: 100 Microns Diameter, 5 Microns Thick Aluminum Disc on $\mathrm{SiO}_{2}$ Back Surface; $18 \mathrm{~J} / \mathrm{cm}^{3}$ Gaussian Pulse, $3 \mathrm{~ns}$ FWHM. Fringe of Pressure (Mbars), Time $=22$ ns.

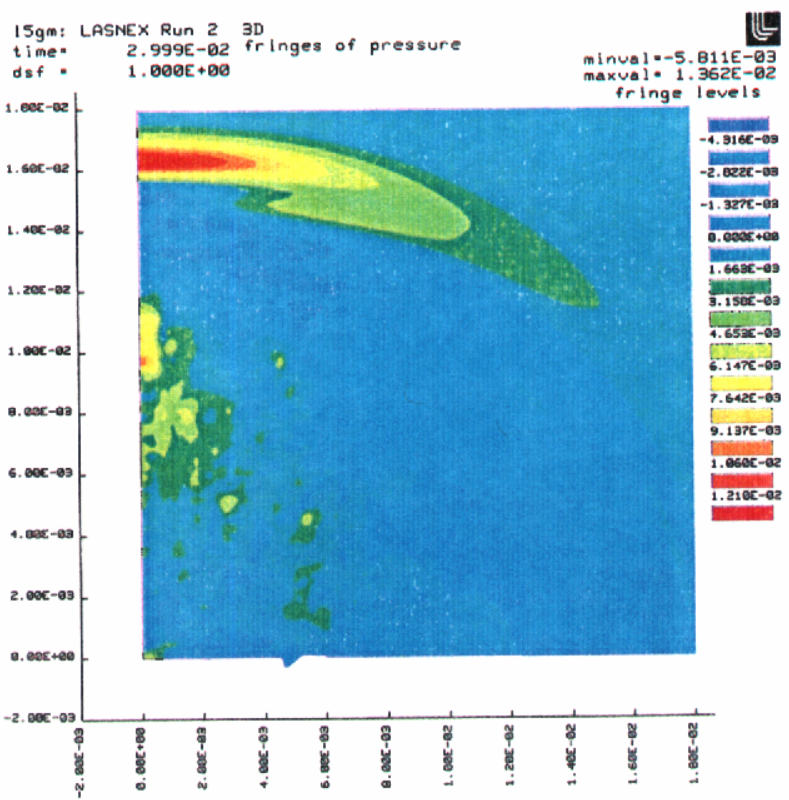

Figure 32: 100 Microns Diameter, 5 Microns Thick Aluminum Disc on $\mathrm{SiO}_{2}$ Back Surface; $18 \mathrm{~J} / \mathrm{cm}^{3}$ Gaussian Pulse, $3 \mathrm{~ns}$ FWHM. Fringe of Pressure (Mbars), Time $=30 \mathrm{~ns}$. 


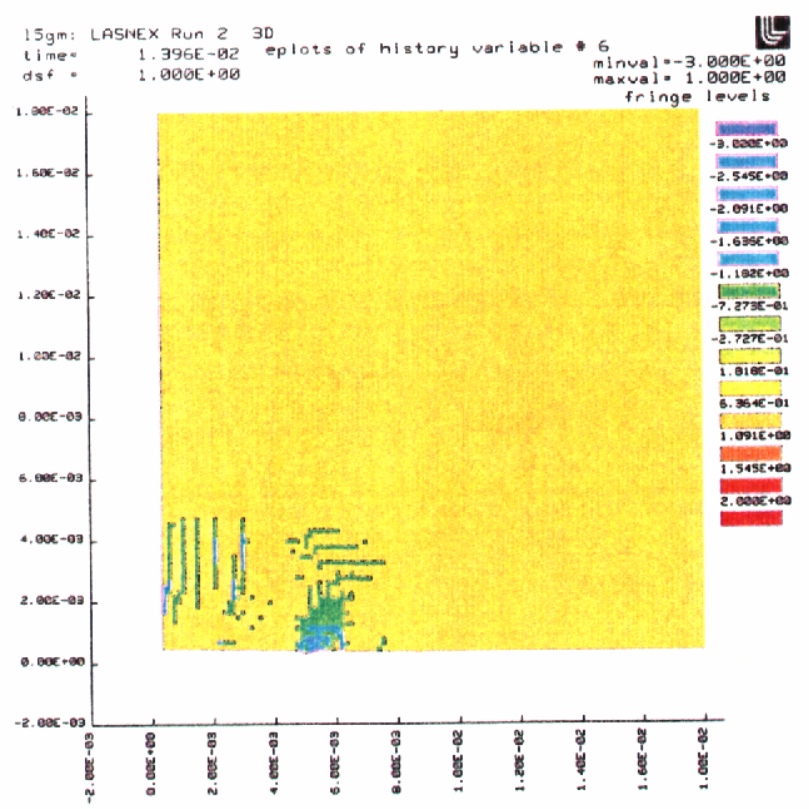

Figure 33: 100 Microns Diameter, 5 Microns Thick Aluminum Disc on $\mathrm{SiO}_{2}$ Back Surface; $18 \mathrm{~J} / \mathrm{cm}^{3}$ Gaussian Pulse, $3 \mathrm{~ns}$ FWHM. Fringe of Damage, Time = 14 ns.

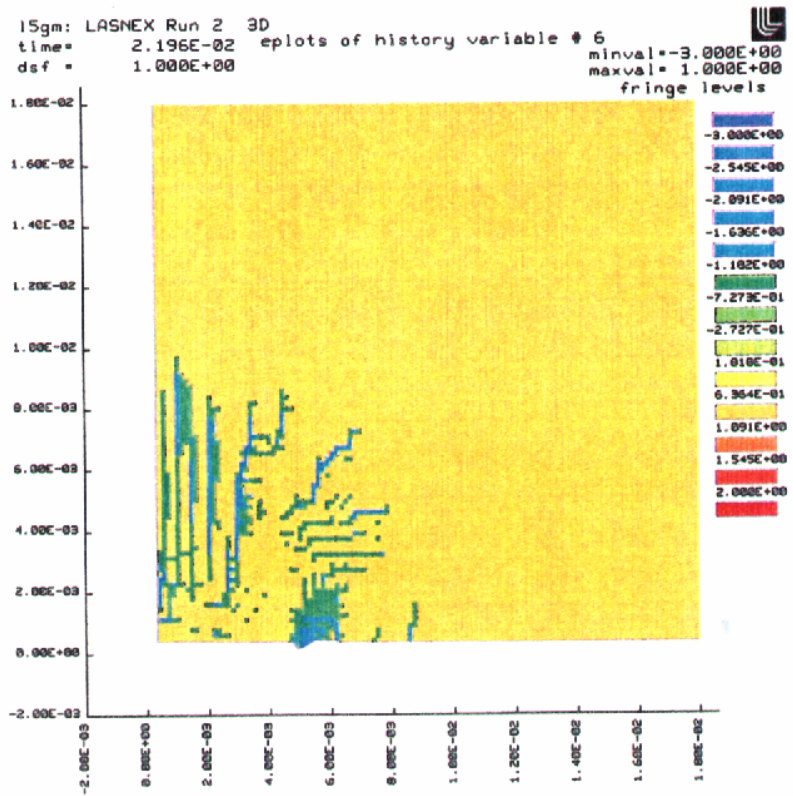

Figure 34: 100 Microns Diameter, 5 Microns Thick Aluminum Disc on $\mathrm{SiO}_{2}$ Back Surface; $18 \mathrm{~J} / \mathrm{cm}^{3}$ Gaussian Pulse, $3 \mathrm{~ns}$ FWHM. Fringe of Damage, Time $=$ 22 ns. 


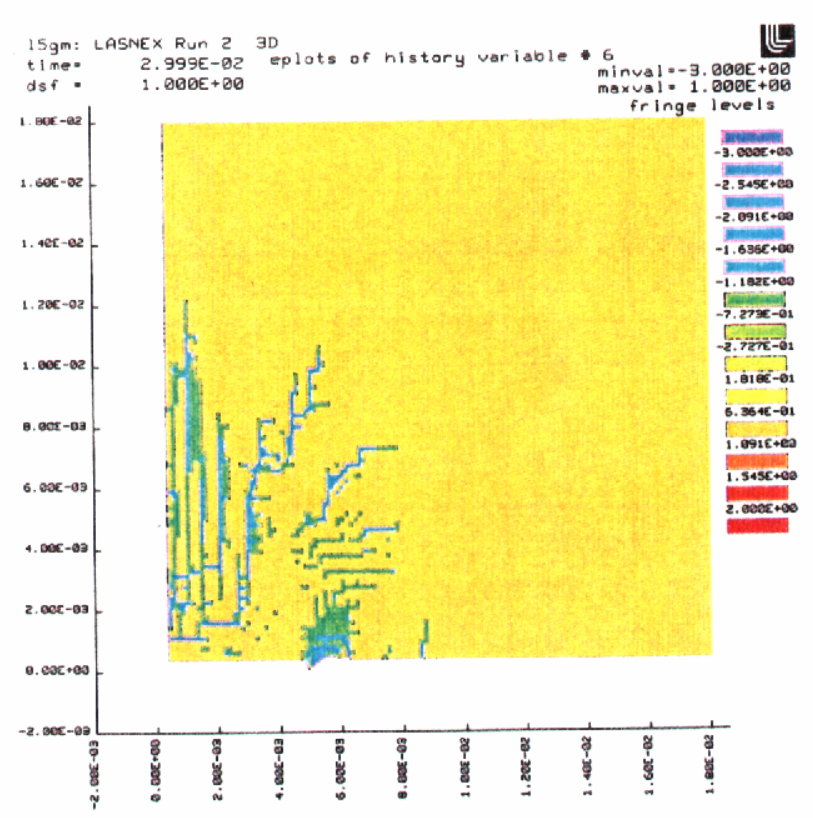

Figure 35: 100 Microns Diameter, 5 Microns Thick Aluminum Disc on $\mathrm{SiO}_{2}$ Back Surface; $18 \mathrm{~J} / \mathrm{cm}^{3}$ Gaussian Pulse, 3 ns FWHM. Fringe of Damage, Time = $30 \mathrm{~ns}$.

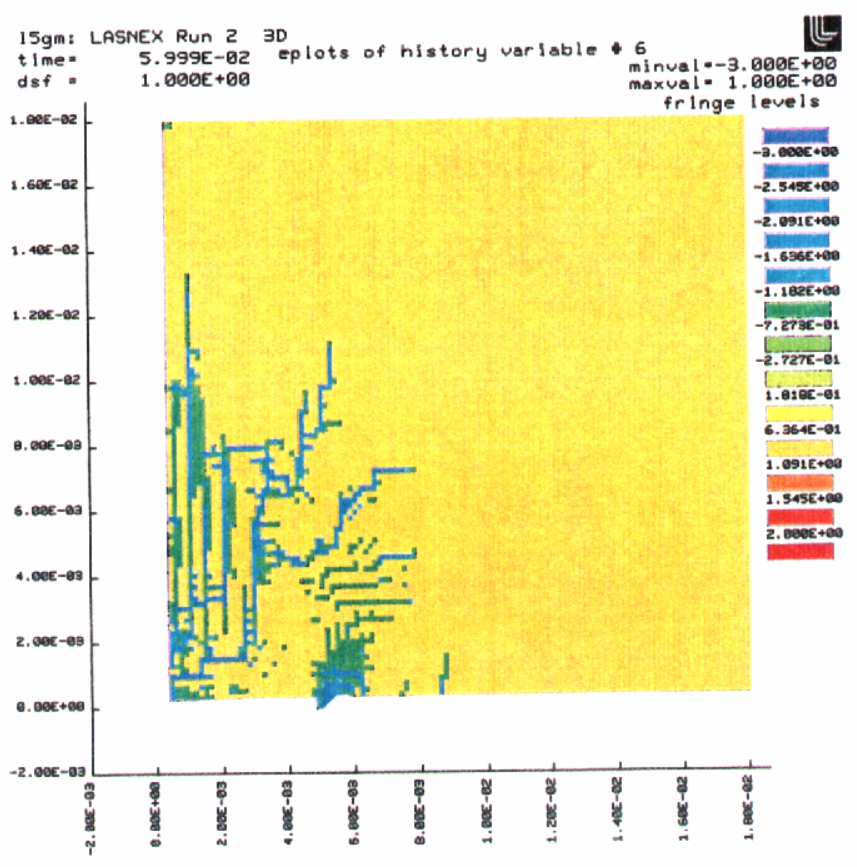

Figure 36: 100 Microns Diameter, 5 Microns Thick Aluminum Disc on $\mathrm{SiO}_{2}$ Back Surface; $18 \mathrm{~J} / \mathrm{cm}^{3}$ Gaussian Pulse, $3 \mathrm{~ns}$ FWHM. Fringe of Damage, Time = $60 \mathrm{~ns}$. 
UCRL-ID-128908

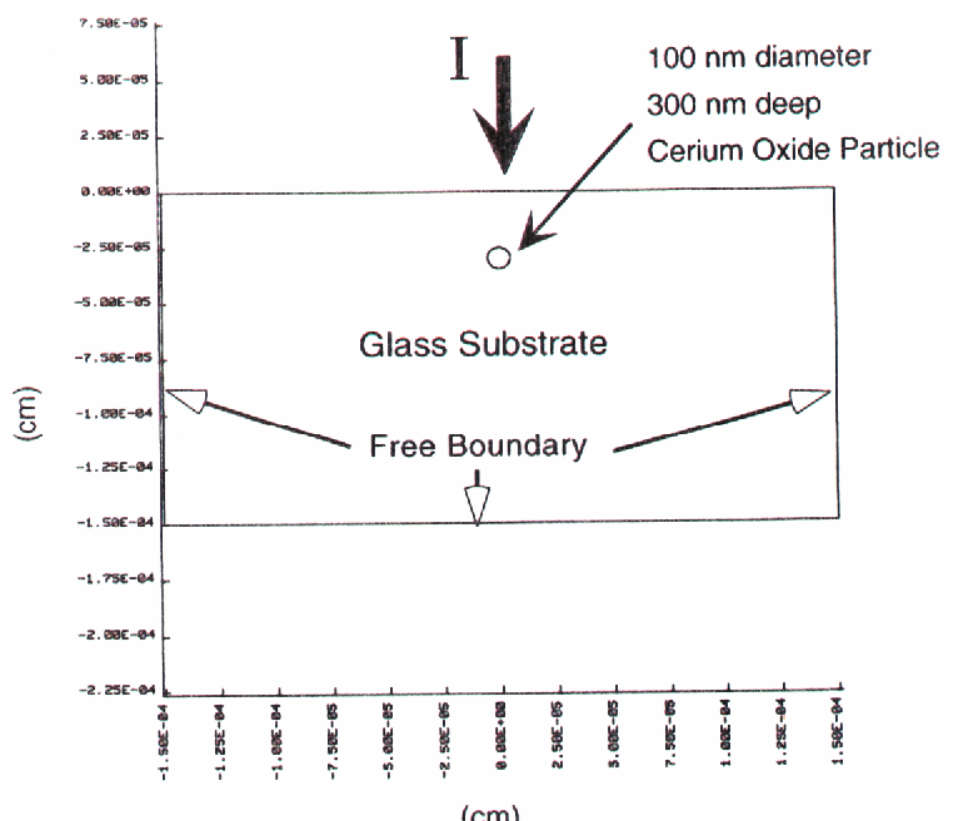

Figure 37: 100 Nanometers Diameter Cerium Oxide Particle, 300 Nanometers Deep in $\mathrm{SiO}_{2}$ Substrate; Geometry Outline, Time $=0$ nanoseconds.

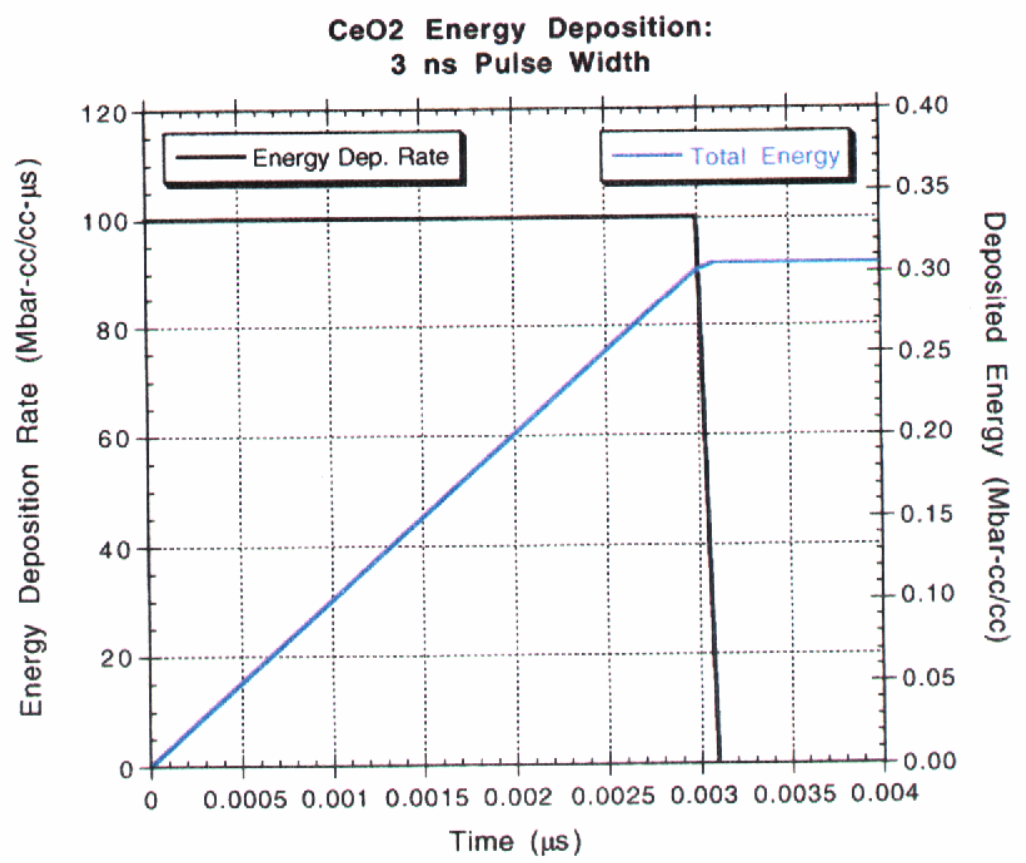

Figure 38: 100 Nanometers Diameter Cerium Oxide Particle, 300 Nanometers Deep in $\mathrm{SiO}_{2}$ Substrate; Energy Deposition Rate and Total Energy in $\mathrm{CeO} 2$. 


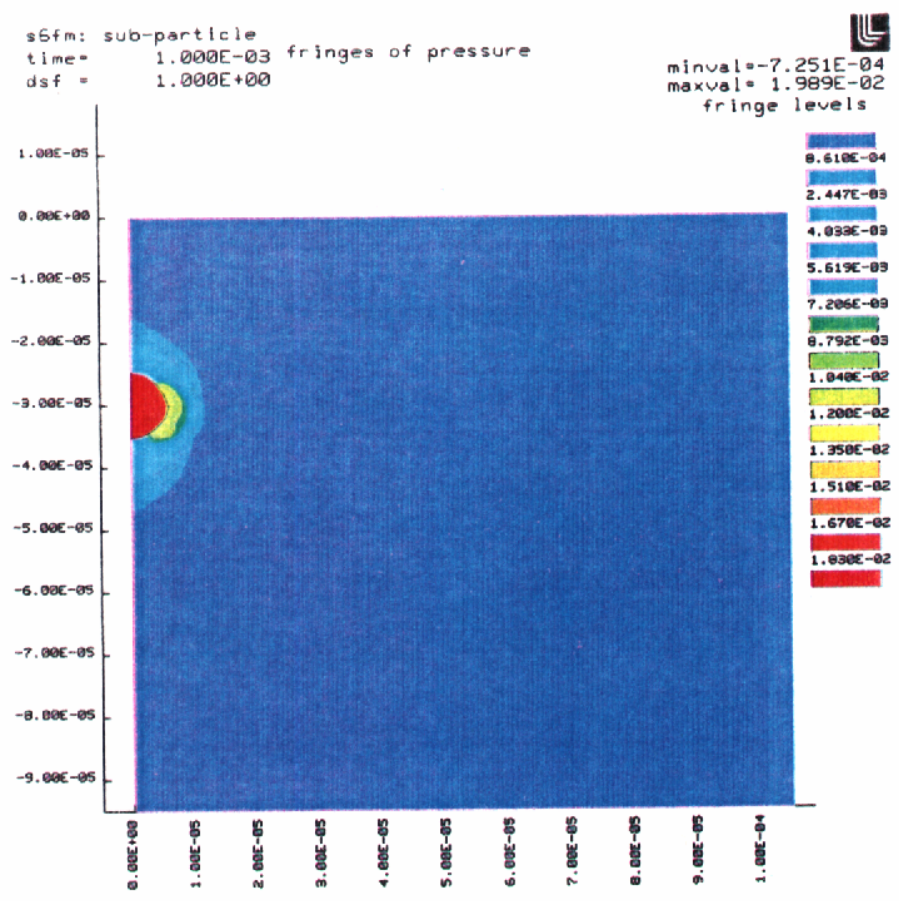

Figure 39: 100 Nanometers Diameter Cerium Oxide Particle, 300 Nanometers Deep in $\mathrm{SiO}_{2}$ Substrate; Fringe of Pressure (Mbars), Time $=1.0$ nanoseconds.

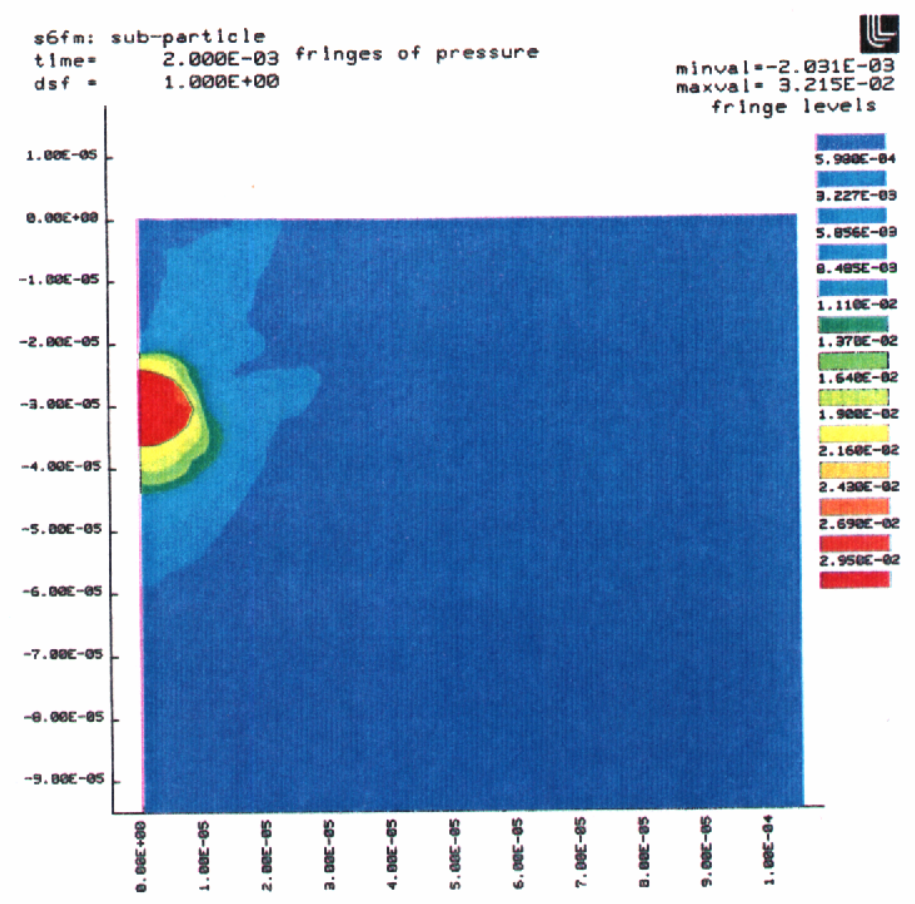

Figure 40: 100 Nanometers Diameter Cerium Oxide Particle, 300 Nanometers Deep in $\mathrm{SiO}_{2}$ Substrate; Fringe of Pressure (Mbars), Time $=2.0$ nanoseconds. 


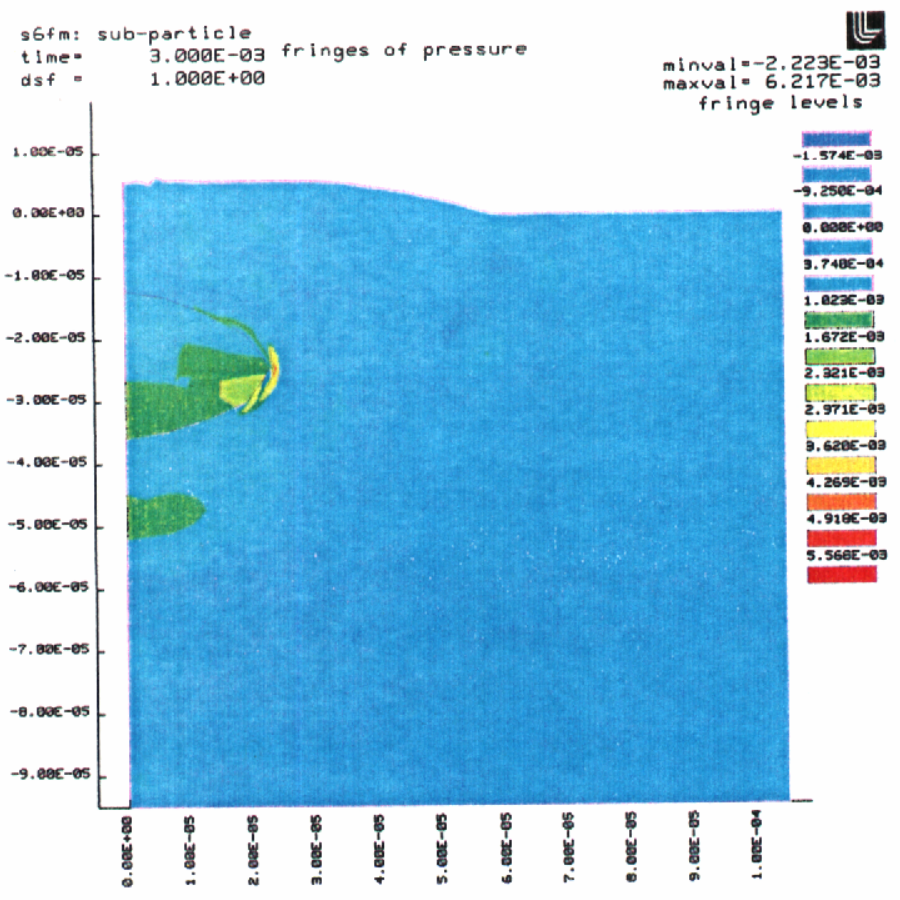

Figure 41: 100 Nanometers Diameter Cerium Oxide Particle, 300 Nanometers Deep in $\mathrm{SiO}_{2}$ Substrate; Fringe of Pressure (Mbars), Time $=3.0$ nanoseconds.

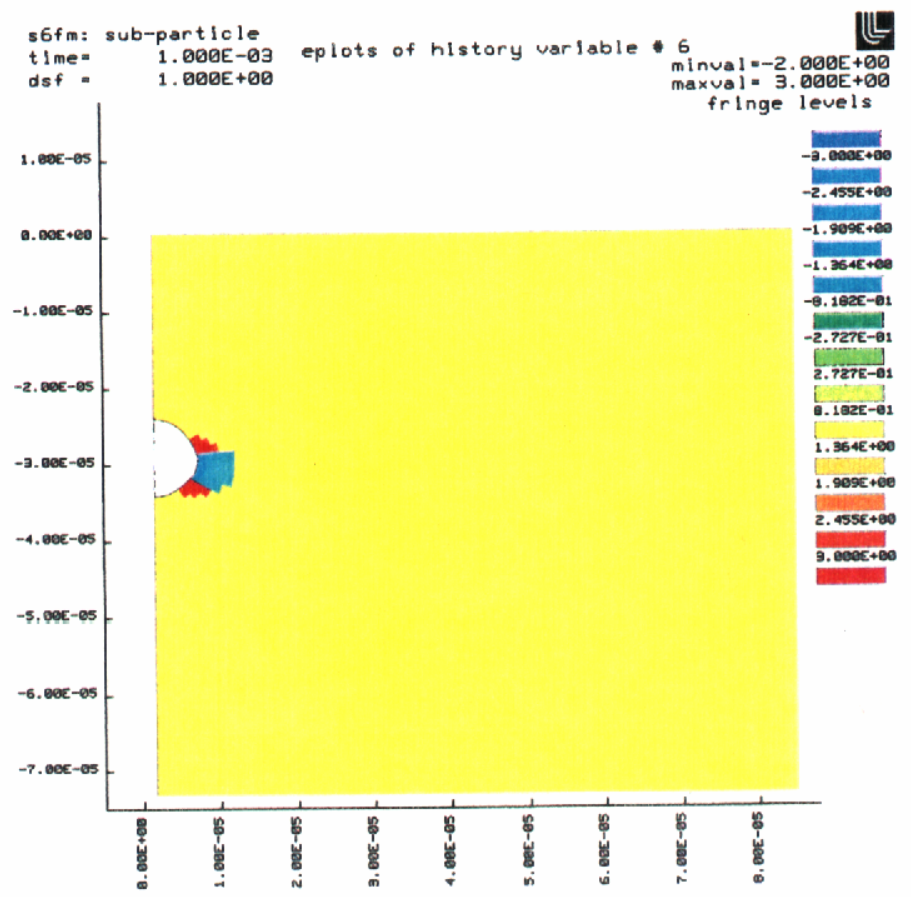

Figure 42: 100 Nanometers Diameter Cerium Oxide Particle, 300 Nanometers Deep in $\mathrm{SiO}_{2}$ Substrate; Fringe of Damage, Time $=1.0$ nanoseconds. 


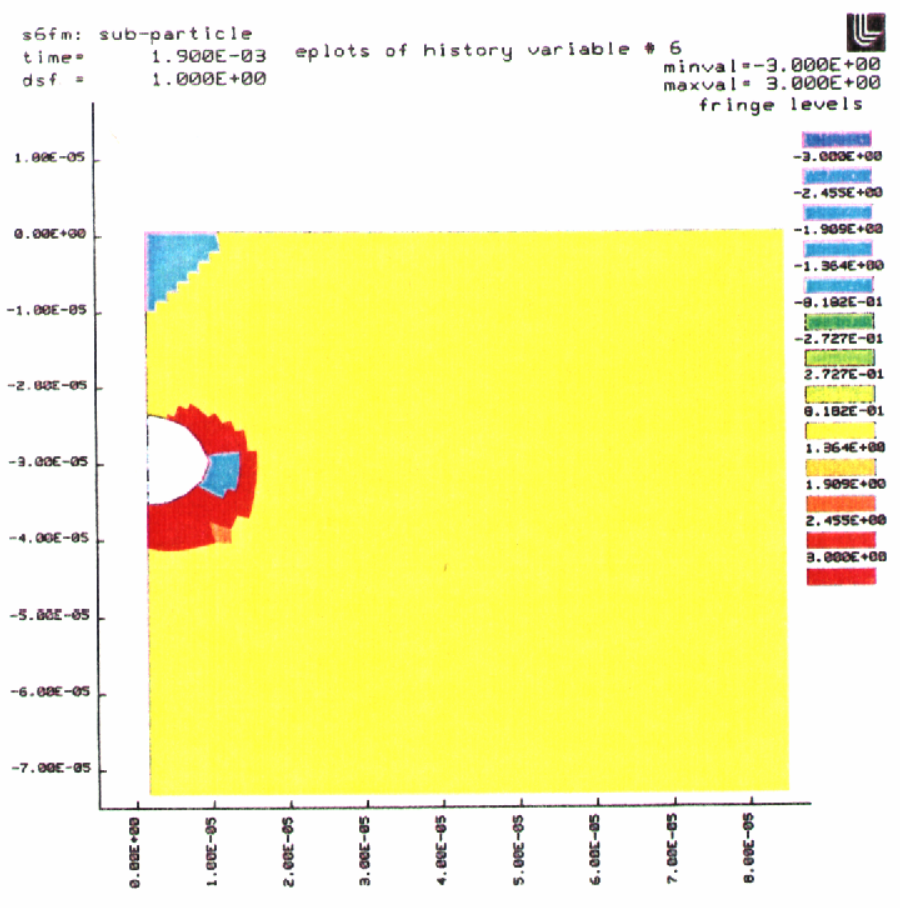

Figure 43: 100 Nanometers Diameter Cerium Oxide Particle, 300 Nanometers Deep in $\mathrm{SiO}_{2}$ Substrate; Fringe of Damage, Time $=1.9$ nanoseconds.

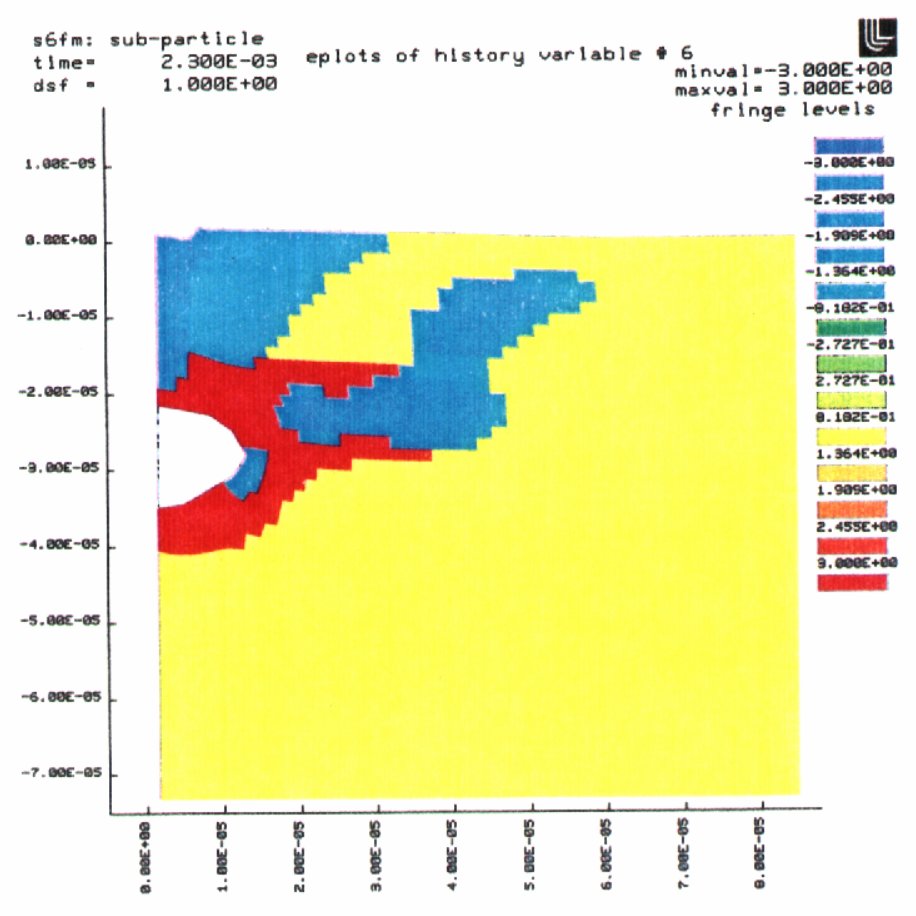

Figure 44: 100 Nanometers Diameter Cerium Oxide Particle, 300 Nanometers Deep in $\mathrm{SiO}_{2}$ Substrate; Fringe of Damage, Time $=2.3$ nanoseconds. 


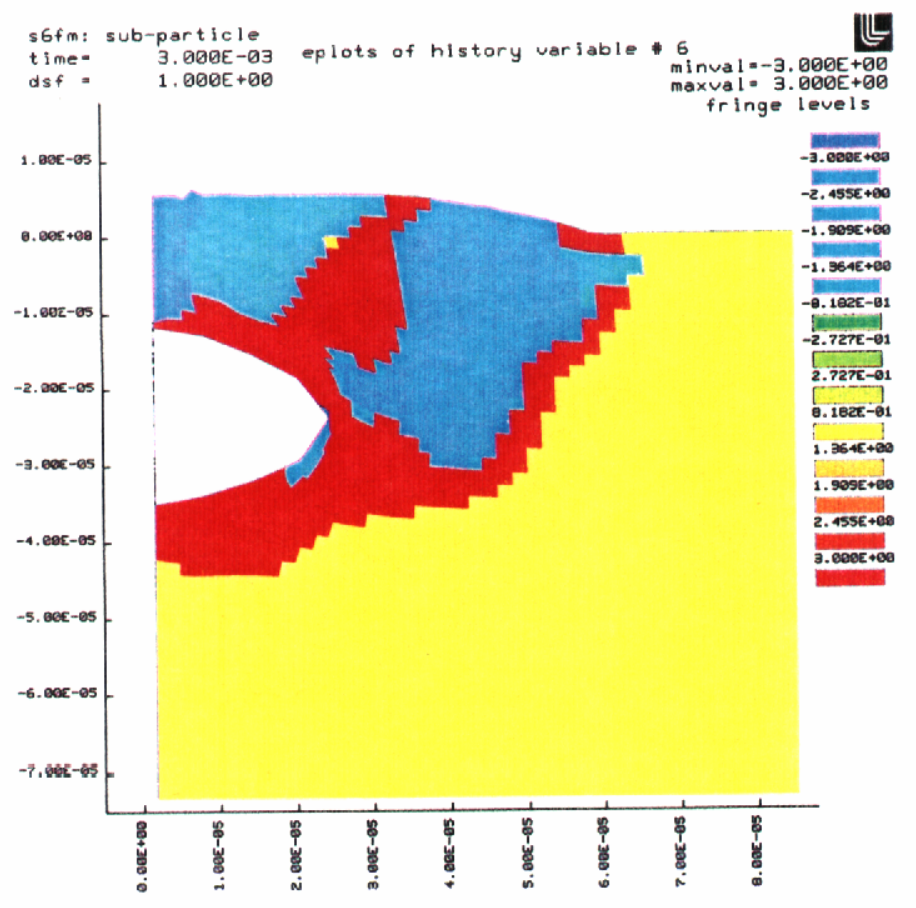

Figure 45: 100 Nanometers Diameter Cerium Oxide Particle, 300 Nanometers Deep in $\mathrm{SiO}_{2}$ Substrate; Fringe of Damage, Time $=3.0$ nanoseconds.

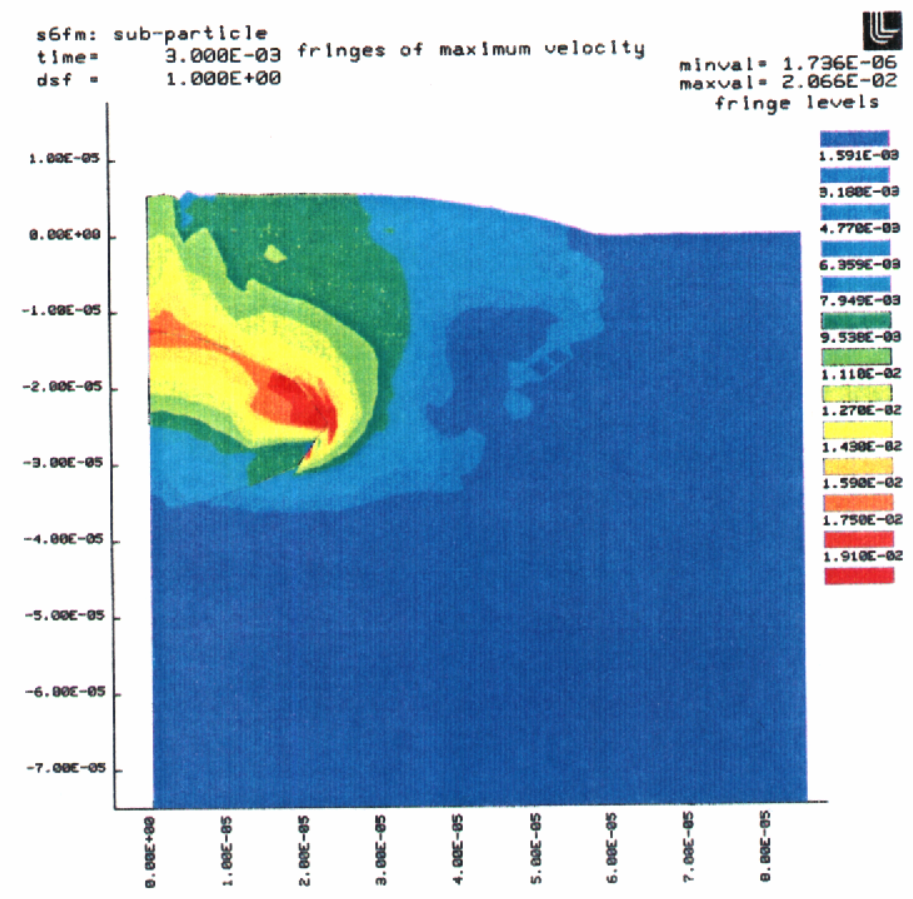

Figure 46: 100 Nanometers Diameter Cerium Oxide Particle, 300 Nanometers Deep in $\mathrm{SiO}_{2}$ Substrate; Fringe of Speed $(\mathrm{cm} / \mu \mathrm{s})$, Time $=3.0$ nanoseconds. 


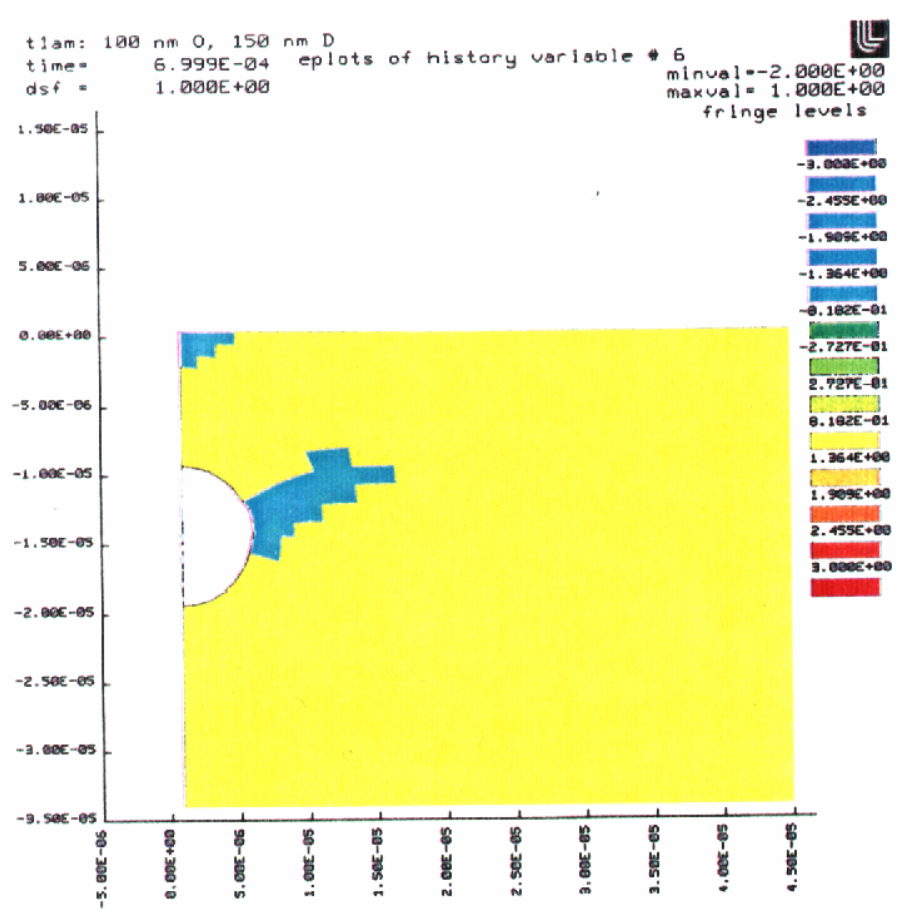

Figure 47: 100 Nanometers Diameter Cerium Oxide Particle, 150 Nanometers Deep in $\mathrm{SiO}_{2}$ Substrate; Fringe of Damage, Time $=0.7$ nanoseconds.

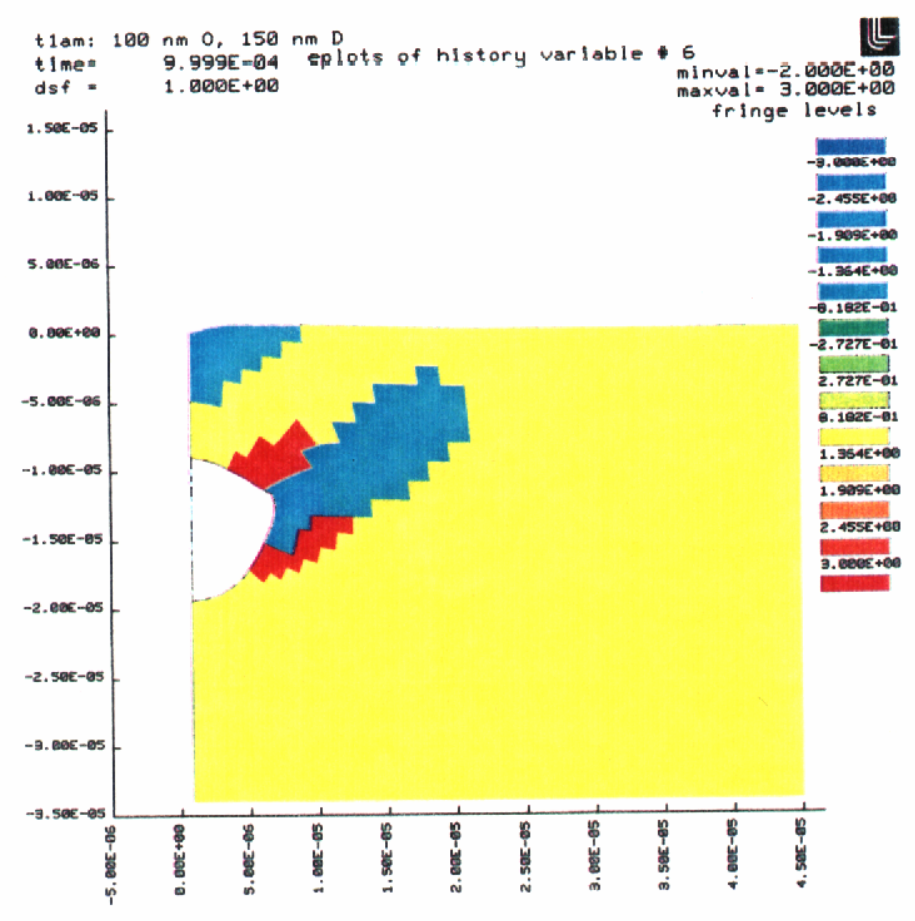

Figure 48: 100 Nanometers Diameter Cerium Oxide Particle, 150 Nanometers Deep in $\mathrm{SiO}_{2}$ Substrate; Fringe of Damage, Time $=1.0$ nanoseconds. 


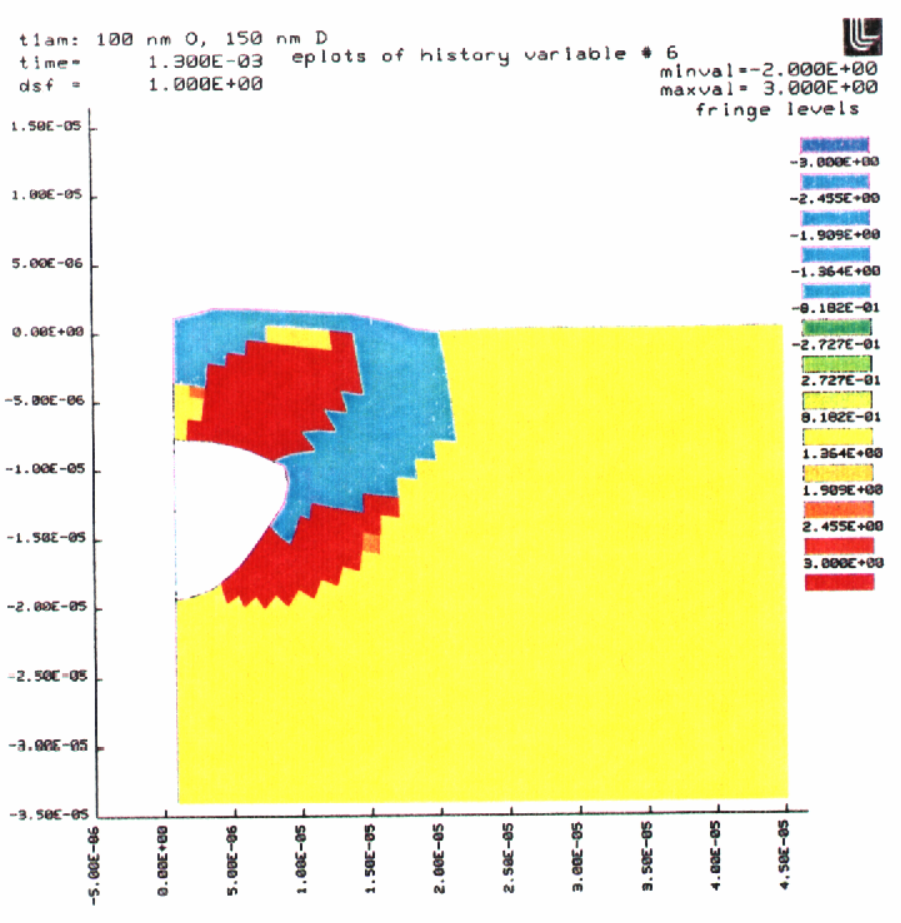

Figure 49: 100 Nanometers Diameter Cerium Oxide Particle, 150 Nanometers Deep in $\mathrm{SiO}_{2}$ Substrate; Fringe of Damage, Time $=1.3$ nanoseconds.

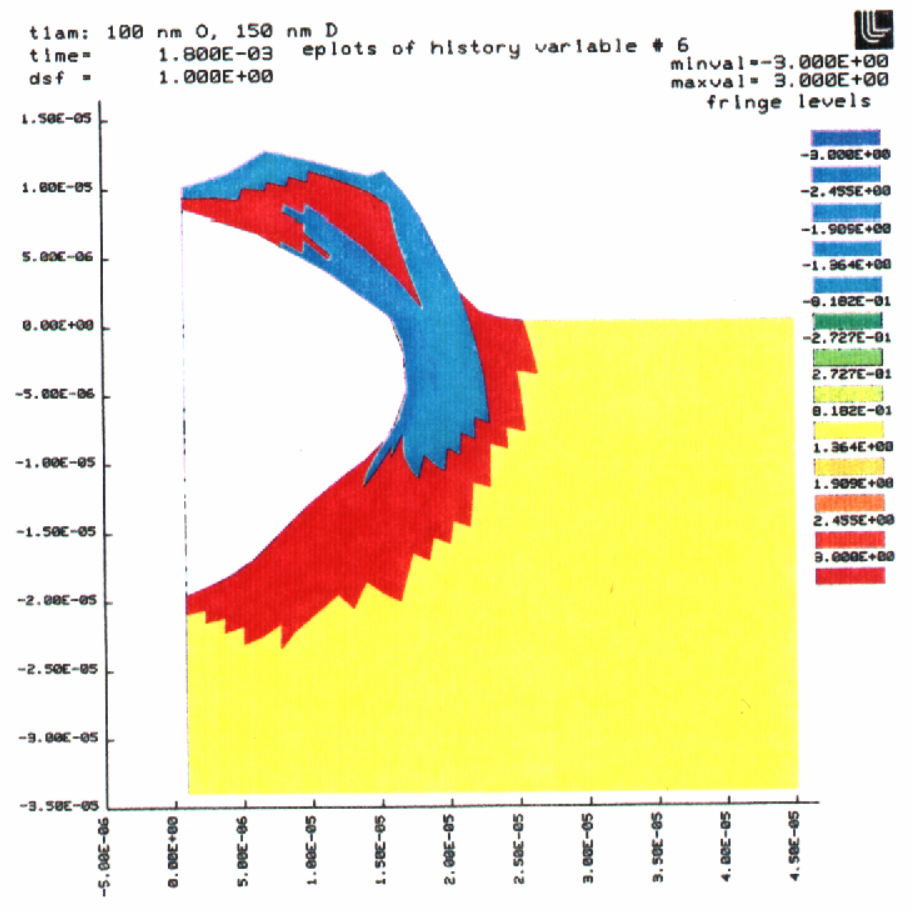

Figure 50: 100 Nanometers Diameter Cerium Oxide Particle, 150 Nanometers Deep in $\mathrm{SiO}_{2}$ Substrate; Fringe of Damage, Time $=1.8$ nanoseconds. 


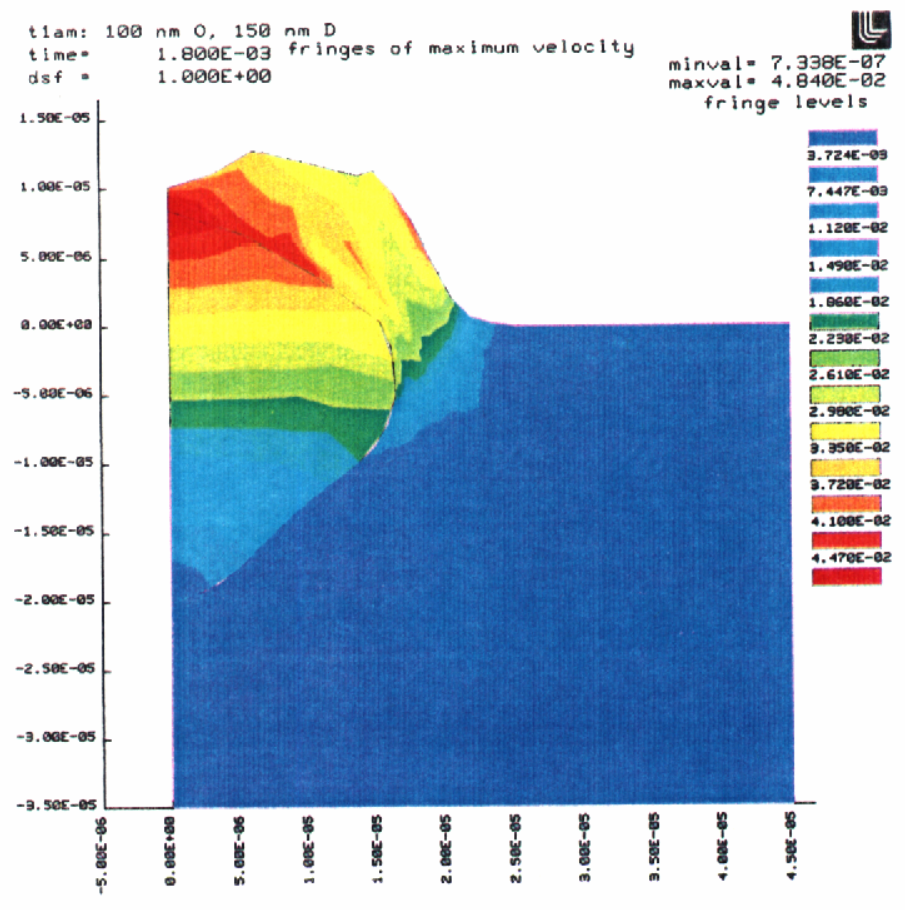

Figure 51: 100 Nanometers Diameter Cerium Oxide Particle, 150 Nanometers Deep in $\mathrm{SiO}_{2}$ Substrate; Fringe of Speed $(\mathrm{cm} / \mu \mathrm{s})$, Time $=1.8$ nanoseconds. 


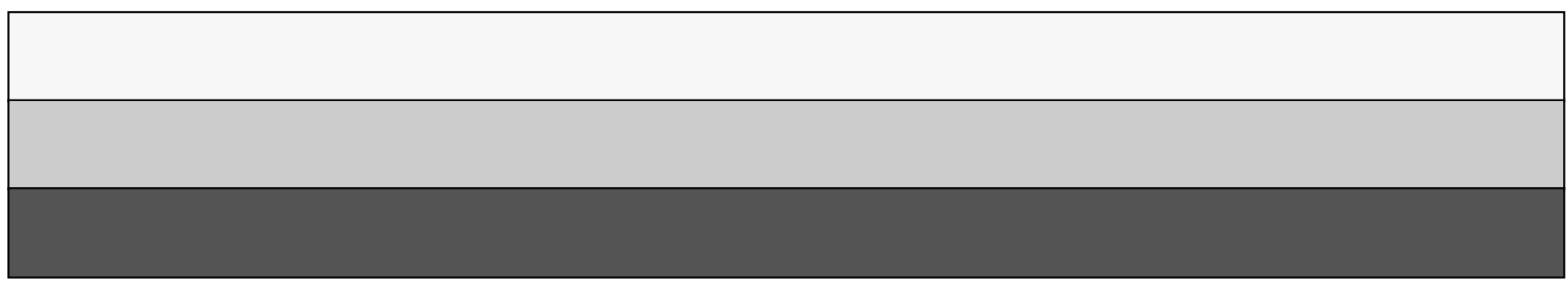

